\title{
The universal properties of Teichmüller spaces
}

\author{
Vladimir Markovic and Dragomir Šarić
}

\begin{abstract}
We discuss universal properties of general Teichmüller spaces. Our topics include the Teichmüller metric and Kobayashi metric, extremality and unique extremality of quasiconformal mappings, biholomorphic maps between Teichmüller space, earthquakes and Thurston boundary.
\end{abstract}

\section{Introduction}

Today, Teichmüller theory is a substantial area of mathematics that has interactions with many other subjects. The bulk of this theory is focused on studying Teichmüller spaces of finite type Riemann surfaces. In this article we survey the theory that investigates all Teichmüller spaces regardless of their dimension. We aim to present theorems (old and recent) that illustrate universal properties of Teichmüller spaces.

Teichmüller spaces of finite type Riemann surfaces are finite-dimensional complex manifolds with rich geometric structures. Teichmüller spaces of infinite type Riemann surfaces are infinite-dimensional Banach manifolds whose geometry differs significantly from the finite case. However, some statements hold for both finite and infinite cases. The intent is to describe these universal properties of all Teichmüller spaces and to point out to differences between finite and infinite cases when these are well understood.

The following is a brief list of topics covered. In the second section we briefly introduce quasiconformal maps and mention their basic properties. Then we proceed to give the analytic definition of Teichmüller spaces, regardless whether the underlying Riemann surface is of finite or infinite type. Then we define the Teichmüller metric and introduce the complex structure on Teichmüller spaces. We discuss the Kobayashi metric, the tangent

1991 Mathematics Subject Classification. 30F60.

Key words and phrases. universal Teichmüller space, quasiconformal maps, quasisymmetric maps, extremal maps, biholomorphic maps, earthquakes, Thurston's boundary.

The second author was partially supported by PSC-CUNY grant PSCREG-39-386.

(C)2009 International Press 
space and the barycentric extensions. In the third section we consider the geometry of Teichmüller spaces. We discuss the Reich-Strebel inequality and the Teichmüller theorem, the Finsler structure, the universal Teichmüller space, the extremal and uniquely extremal quasiconformal maps. In section four we consider biholomorphic maps between Teichmüller spaces and give a short proof that the modular group is the full group of biholomorphic maps of the Teichmüller space of a finite surface following [19]. In section five we consider earthquakes and bendings on infinite surfaces, and we introduce the Thurston boundary for general Teichmüller spaces. We refer the reader to $[\mathbf{6 1}]$ for the discussion of the universal properties of the Weil-Petersson metric.

\section{The Teichmüller space: definition, the Teichmüller and Kobayashi metric, the complex structure and the barycentric extension}

We start with basic definitions. Let $M$ be a Riemann surface. The Uniformization theorem states that the universal covering of $M$ is either the complex plane $\mathbf{C}$, the Riemann sphere $\widehat{\mathbf{C}}=\mathbf{C} \cup\{\infty\}$ or the upper half-plane $\mathbf{H}$. The complex plane $\mathbf{C}$, the once punctured complex plane $\mathbf{C} \backslash\{0\}$ and the torus $T$ is the short list of Riemann surfaces covered by $\mathbf{C}$. The Riemann sphere $\widehat{\mathbf{C}}$ is the only Riemann surface whose universal covering is $\widehat{\mathbf{C}}$. The Teichmüller spaces of $\widehat{\mathbf{C}}, \mathbf{C}$ and $\mathbf{C} \backslash\{0\}$ consist of a single point, while the Teichmüller space of the torus $T$ is biholomorphic to the upper half-plane $\mathbf{H}$ and isometric to the hyperbolic plane (for example, see [38]). Thus, the Teichmüller space of a Riemann surface $M$ whose universal covering is either $\widehat{\mathbf{C}}$ or $\mathbf{C}$ is well understood. We focus on the case when $\mathbf{H}$ is the universal covering.

A quasiconformal map $f: \mathbf{H} \rightarrow \mathbf{H}$ is an orientation preserving homeomorphism which is absolutely continuous on lines and which satisfies $\|\bar{\partial} f / \partial f\|_{\infty}<1$. The Beltrami coefficient $\mu=\bar{\partial} f / \partial f$ of a quasiconformal map $f$ is defined almost everywhere and it satisfies $\|\mu\|_{\infty}<1$. The quasiconformal constant $K(f)$ of $f$ is given by $K(f)=\frac{1+\|\mu\|_{\infty}}{1-\|\mu\|_{\infty}}$. Note that $\|\mu\|_{\infty}<1$ if and only if $K(f)<\infty$.

Given a measurable function $\mu$ on $\mathbf{H}$ such that $\|\mu\|_{\infty}<1$, there exists a quasiconformal map $f: \mathbf{H} \rightarrow \mathbf{H}$ such that $\mu=\bar{\partial} f / \partial f$. The quasiconformal map $f$ is unique up to post-composition by a Möbius map preserving $\mathbf{H}$ $($ see $[4])$.

2.1. Definition of the Teichmüller space. From now on we assume that the universal covering of a Riemann surface $M$ is the upper half-plane $\mathbf{H}$. We identify the hyperbolic plane with the upper half-plane $\mathbf{H}$ equipped with the metric $\rho(z)=\frac{|d z|}{2 y}$, where $z=x+i y \in \mathbf{H}$. The universal covering 
map $\pi: \mathbf{H} \rightarrow M$ induces a hyperbolic metric on $M$. The Riemann surface $M$ is said to be hyperbolic.

Let $G$ be a Fuchsian group acting on the upper half-plane $\mathbf{H}$ such that $M$ is conformal and isometric to $\mathbf{H} / G$. (The group $G$ is unique up to conjugation by a Möbius map fixing $\mathbf{H}$.) Let $P S L_{2}(\mathbf{R})$ denote the subgroup of the Möbius group which fixes the upper half-plane $\mathbf{H}$.

Definition 2.1. Let $M$ be a hyperbolic surface. Let $G$ be a Fuchsian group such that $M$ is isomorphic to $\mathbf{H} / G$. The Teichmüller space $T(M)$ of $M$ consists of equivalence classes of quasiconformal maps $f: \mathbf{H} \rightarrow \mathbf{H}$ which satisfy the following condition

$$
f \circ \gamma \circ f^{-1} \in P S L_{2}(\mathbf{R}),
$$

for all $\gamma \in G$. Two such quasiconformal maps $f_{1}, f_{2}: \mathbf{H} \rightarrow \mathbf{H}$ are equivalent if their extensions to the extended real line $\widehat{\mathbf{R}}=\mathbf{R} \cup\{\infty\}$ agree up to a postcomposition by a Möbius map, i.e., $f_{1}$ is equivalent to $f_{2}$ if $\left.f_{1}\right|_{\widehat{\mathbf{R}}}=\left.\beta \circ f_{2}\right|_{\widehat{\mathbf{R}}}$ for some $\beta \in P S L_{2}(\mathbf{R})$.

REMARK. The definition of $T(M)$ depends on the choice of the Fuchsian group $G$. Since $G$ is unique up to conjugation by an element of $P S L_{2}(\mathbf{R})$ it is easy to check that all subsequent definitions are independent of this choice. We denote by $[f]$ the equivalence class of the quasiconformal map $f: \mathbf{H} \rightarrow \mathbf{H}$ satisfying the invariance property (1). Then $[f] \in T(M)$.

REMARK. In the above definition, we could replace quasiconformal maps of $\mathbf{H}$ with quasisymmetric maps of $\widehat{\mathbf{R}}$ which satisfy the invariance property (1) on $\widehat{\mathbf{R}}$. This follows from the Douady-Earle barycentric extension [11].

REMARK. The map $f: \mathbf{H} \rightarrow \mathbf{H}$ which satisfies the invariance property (1) projects to a quasiconformal map $\hat{f}: M \rightarrow M_{1}$, where $M=\mathbf{H} / G$ and $M_{1}$ is a Riemann surface whose covering Fuchsian group is $f G f^{-1}$. The condition that $\left.f_{1}\right|_{\widehat{\mathbf{R}}}=\left.\beta \circ f_{2}\right|_{\widehat{\mathbf{R}}}$ is equivalent to the property that the projections $\hat{f}_{1}$ and $\hat{f}_{2}$ map $M$ onto the same surface and that they are isotopic through a bounded quasiconformal isotopy. The last statement was proved by Earle and McMullen [21] using the Douady-Earle extension.

2.2. The Teichmüller metric. The Teichmüller space $T(M)$ carries a natural metric defined as follows.

Definition 2.2. Let $[f],[g] \in T(M)$. The Teichmüller distance between $[f]$ and $[g]$ is given by

$$
d([f],[g])=\inf _{g_{1} \in[g], f_{1} \in[f]} \frac{1}{2} \log K\left(g_{1} \circ f_{1}^{-1}\right) .
$$

It is easy to check that this distance is in fact a metric. The space $(T(M), d)$ is a complete and non-compact metric space. 
2.3. The complex structure on the Teichmüller space. The Teichmüller space is equipped with a natural complex structure as follows. Let $[f] \in T(M)$ and let $\mu=\bar{\partial} f / \partial f$ be the Beltrami coefficient of $f$. Then $\|\mu\|_{\infty}<1$ and

$$
\mu(z)=\mu(\gamma(z)) \frac{\overline{\gamma^{\prime}(z)}}{\gamma^{\prime}(z)}
$$

for $z \in \mathbf{H}$ and $\gamma \in G$. Let $\mathbf{L}=\mathbf{C} \backslash \overline{\mathbf{H}}$ be the lower half-plane. Given a Beltrami coefficient $\mu$ on $\mathbf{H}$ which satisfies (2), we define the Beltrami coefficient $\widehat{\mu}$ on $\widehat{\mathbf{C}}$ by $\widehat{\mu}(z)=\mu(z)$ for $z \in \mathbf{H}$, and $\widehat{\mu}(z)=0$ for $z \in \mathbf{L}$. Then $\widehat{\mu}$ satisfies $(2)$ for all $z \in \widehat{\mathbf{C}}$. There exists a quasiconformal map $f: \widehat{\mathbf{C}} \rightarrow \widehat{\mathbf{C}}$ whose Beltrami coefficient is $\widehat{\mu}$ (see $[4]$ for the solution of the Beltrami equation $\bar{\partial} f=\widehat{\mu} \cdot \partial f$ ). Moreover, $f$ is unique up to a post-composition by a Möbius map of the Riemann sphere $\widehat{\mathbf{C}}$, it is conformal in $\mathbf{L}$ and it satisfies the invariance relation (1) in $\widehat{\mathbf{C}}$. Denote this map by $f^{\widehat{\mu}}$.

Let $g$ be a holomorphic map defined in a domain on $\widehat{\mathbf{C}}$. Then the Schwarzian derivative $S(g)$ of $g$ is given by

$$
S(g)=\frac{g^{\prime \prime \prime}}{g^{\prime}}-\frac{3}{2}\left(\frac{g^{\prime \prime}}{g^{\prime}}\right)^{2} .
$$

We recall that the Schwarzian derivative measures by how much a holomorphic map distorts cross-ratios of four points (see [31, Section 6.1]). In particular, the Schwarzian derivative of a Möbius map is zero.

If we apply the Schwarzian derivative to $f^{\widehat{\mu}}$ in $\mathbf{L}$, then we obtain a holomorphic map $S\left(f^{\widehat{\mu}}\right)$ on $\mathbf{L}$ which satisfies

$$
\left(S\left(f^{\widehat{\mu}}\right) \circ \gamma\right)(z)\left(\gamma^{\prime}(z)\right)^{2}=S\left(f^{\widehat{\mu}}\right)(z)
$$

and

$$
\sup _{z \in \mathbf{L}}\left|S\left(f^{\widehat{\mu}}\right)(z) \rho_{\mathbf{L}}^{-2}(z)\right|<\infty
$$

for $z \in \mathbf{L}$ and $\gamma \in G$, where $\rho_{\mathbf{L}}(z)=\frac{|d z|}{2|y|}$ is the Poincaré metric on $\mathbf{L}$ (see [6], $[26]$ or $[31])$.

Let $B_{\mathbf{L}}(G)$ be the Banach space of all holomorphic maps $\psi: \mathbf{L} \rightarrow \mathbf{C}$ which satisfy $\psi(\gamma(z)) \gamma^{\prime}(z)^{2}=\psi(z)$, for $z \in \mathbf{L}$ and $\gamma \in G$, and $\left\|\psi \rho_{\mathbf{L}}^{-2}\right\|_{\infty}=$ $\sup _{z \in \mathbf{L}}\left|\psi(z) \rho_{\mathbf{L}}^{-2}(z)\right|<\infty$, where $\left\|\psi \rho_{\mathbf{L}}^{-2}\right\|_{\infty}$ is the norm on $B_{\mathbf{L}}(G)$. Note that $\mathcal{S}\left(f^{\hat{\mu}}\right) \in B_{\mathbf{L}}(G)$.

If a quasiconformal map $f: \mathbf{H} \rightarrow \mathbf{H}$ satisfies the invariance property (1), then the Beltrami coefficient $\mu=\bar{\partial} f / \partial f$ of $f$ satisfies the invariance property (2) (and $\|\mu\|_{\infty}<1$ ). Conversely, given measurable $\mu: \mathbf{H} \rightarrow \mathbf{C}$ such that $\|\mu\|_{\infty}<1$ and (2) holds for $\mu$, then there exists a quasiconformal map $f: \mathbf{H} \rightarrow \mathbf{H}$ whose Beltrami coefficient is $\mu$ and which satisfies (1) (see [4]). 
The map $f$ is unique up to post-composition by an element of $P S L_{2}(\mathbf{R})$. (Note that $[\beta \circ f]=[f]$ for $\beta \in P S L_{2}(\mathbf{R})$ by the definition of the Teichmüller equivalence.)

Let $L^{\infty}(G)$ be the Banach space of all measurable essentially bounded functions $\mu$ on $\mathbf{H}$ which satisfy the property (2). Let $U(G)$ be the open unit ball in $L^{\infty}(G)$, namely $U(G)=\left\{\mu \in L^{\infty}(G):\|\mu\|_{\infty}<1\right\}$. Then we have the Schwarzian derivative map

$$
\mathcal{S}: U(G) \rightarrow B_{\mathbf{L}}(G)
$$

given by $\mathcal{S}(\mu)=S\left(f^{\widehat{\mu}}\right)$, where $\widehat{\mu}=\mu$ on $\mathbf{H}$ and $\widehat{\mu}=0$ on $\mathbf{L}$. By the above, the Teichmüller space $T(M)$ is identified with the quotient of $U(G)$, where $\mu_{1}, \mu_{2} \in U(G)$ determine the same point in $T(M)$ if and only if $\left.f^{\mu_{1}}\right|_{\hat{\mathbf{R}}}=$ $\left.\beta \circ f^{\mu_{2}}\right|_{\hat{\mathbf{R}}}$ for some $\beta \in P S L_{2}(\mathbf{R})$. The following result of Bers and AhlforsWeil (see $[\mathbf{3}],[\mathbf{6}],[\mathbf{2 6}],[\mathbf{3 1}$, Section 6], [47]) gives a natural complex Banach manifold structure to general Teichmüller spaces.

Theorem 2.1 (Bers Embedding Theorem and Ahlfors-Weil Section). Let $M$ be a hyperbolic surface and let $G$ be a Fuchsian group such that $\mathbf{H} / G$ is conformal to $M$. The Schwarzian derivative map $\mathcal{S}: U(G) \rightarrow B_{\mathbf{L}}(G)$ induces an injective map

$$
\Phi: T(M) \rightarrow B_{\mathbf{L}}(G)
$$

such that $\Phi(T(M))$ is an open, bounded subset of $B_{\mathbf{L}}(G)$. The map $\Phi$ is a homeomorphism onto its image and defines a global holomorphic chart for $T(S)$. Moreover, given any $\Phi([\mu])=\varphi \in \Phi(T(M)) \subset B_{\mathbf{L}}(G)$ there exists a neighborhood $V_{\varphi}$ of $\varphi$ and a holomorphic map $s_{\varphi}: V_{\varphi} \rightarrow U(G)$ such that $\mathcal{S} \circ s_{\varphi}=i d$ on $V_{\varphi}$ and $s_{\varphi} \circ \mathcal{S}(\mu)=\mu$.

REMARK. The fact that $\mathcal{S}: U(G) \rightarrow B_{\mathbf{L}}(G)$ induces a map on the Teichmüller space $T(M)$ is equivalent to the statement that if $\mu$ and $\mu_{1}$ give rise to two Teichmüller equivalent maps of $\mathbf{H}$ then $S\left(f^{\widehat{\mu}}\right)=S\left(f^{\widehat{\mu}_{1}}\right)$. The fact that the induced map is injective is equivalent to the statement that if $S\left(f^{\widehat{\mu}}\right)=S\left(f^{\widehat{\mu}_{1}}\right)$ then $\mu$ and $\mu_{1}$ are Teichmüller equivalent.

REMARK. Let $\mu$ be in $U(G)$. Then the quasiconformal map $f^{\mu}: \mathbf{H} \rightarrow \mathbf{H}$, whose Beltrami coefficient is $\mu$, conjugates $G$ onto a Fuchsian group $G^{\mu}$. Let $M^{\mu}=\mathbf{H} / G^{\mu}$. There is a natural bijection $T(\mu): T\left(M^{\mu}\right) \rightarrow T(M)$ given by $[g] \mapsto\left[g \circ f^{\mu}\right]$ which is an isometry for the Teichmüller metrics. (The map $T(\mu)$ is called the translation map.) Let $\Phi_{\mu}: T\left(M^{\mu}\right) \rightarrow B\left(G^{\mu}\right)$ be the Bers map for $M^{\mu}$ (see above for the definition). The fact that $\Phi: T(M) \rightarrow B_{\mathbf{L}}(G)$ is a global complex chart is equivalent to the statement that $\Phi \circ T(\mu) \circ$ $\Phi_{\mu}^{-1}: \Phi_{\mu}\left(T\left(M^{\mu}\right)\right) \rightarrow \Phi(T(M))$ is a biholomorphic map. The fact that the map is holomorphic is a direct consequence of the cocycle property for the Schwarzian (for example, see [31, Section 6.4]). 
The asymptotic Teichmüller space $A T(M)$ of a geometrically infinite Riemann surface $M$ is the quotient of the Teichmüller space $T(M)$ as follows. The quasiconformal map $f: M \rightarrow M_{1}$ is said to be asymptotically conformal if for every $\epsilon>0$ there exists a compact set $K \subset M$ such that $\left\|\left.(\bar{\partial} f / \partial f)\right|_{M \backslash K}\right\|_{\infty}<\epsilon$. By the definition, $[f],[g] \in T(M)$ determine the same point in $A T(M)$ if $\left[f \circ g^{-1}\right]$ has an asymptotically conformal representative (see [14], [32]). The Bers map $\Phi: T(M) \rightarrow B_{\mathbf{L}}(G)$ induces the asymptotic Bers map $\bar{\Phi}: A T(M) \rightarrow B_{\mathbf{L}}(G) / B_{\mathbf{L}}^{0}(G)$, where $B_{\mathbf{L}}^{0}(G)$ is the space of all $\psi \in B_{\mathbf{L}}(G)$ which vanish at infinity on $\mathbf{L} / \mathbf{G}$ (i.e., $\psi \in B_{\mathbf{L}}^{0}(G)$ if for every $\epsilon>0$ there exists a compact set $K \subset \mathbf{L} / \mathbf{G}$ such that $\|\left.\psi \rho_{\mathbf{L}}^{-2}\right|_{\mathbf{L} \backslash \tilde{\mathbf{K}}}<\epsilon$, where $\tilde{K}$ is the lift of $K$ to $\mathbf{L}$ ). Earle, Gardiner and Lakic [14] showed that the asymptotic Bers map is a local homeomorphism. Later Earle, Markovic and Sarić $[\mathbf{2 0}]$ proved the following theorem that completed the picture for $A T(M)$.

THEOREM 2.2. Let $M$ be a geometrically infinite Riemann surface and let $G$ be a Fuchsian group such that $M$ is isomorphic to $\mathbf{H} / G$. Then the asymptotic Bers map

$$
\bar{\Phi}: A T(M) \rightarrow B_{\mathbf{L}}(G) / B_{\mathbf{L}}^{0}(G)
$$

is a biholomorphic map onto a bounded open subset of $B_{\mathbf{L}}(G) / B_{\mathbf{L}}^{0}(G)$.

2.4. The Kobayashi metric on the Teichmüller space. On a given complex Banach manifold one can define (in several ways) a natural pseudometric in terms of the underlying complex structure.

Definition 2.3. Let $X$ be a complex Banach manifold and let $T_{X}$ be its complex tangent bundle. Let $\operatorname{Hol}\left(\mathbf{D}_{r}, X\right)$ be the space of holomorphic maps from the disk $\mathbf{D}_{r}$ into $X$. Let $(v, x)$ be the tangent vector at $x \in X$. The Kobayashi pseudo-metric $k_{X}: T_{X} \rightarrow \mathbf{R}$ is given by

(7) $k_{X}(v, x)=\inf \left\{\frac{1}{r} \mid \exists f \in \operatorname{Hol}\left(\mathbf{D}_{r}, X\right): f(0)=x \quad\right.$ and $\left.\quad d f_{0}(\partial / \partial z)=v\right\}$.

It is a well-known fact that the Kobayashi pseudo-metric on a complex manifold $X$ is the largest pseudo-metric such that any map in $\operatorname{Hol}(\mathbf{D}, X)$ is weakly contracting for the Poincaré metric on the unit disk $\mathbf{D}$. This metric is very rarely Riemannian but it has a Finsler structure.

The following theorem is due to Royden [50] in the case of a closed Riemann surfaces. In the case of a geometrically infinite Riemann surfaces it is proved by Gardiner [27].

Theorem 2.3. Let $M$ be a hyperbolic Riemann surface. Then the Teichmüller metric is equal to the Kobayashi metric on the Teichmüller space $T(M)$ of $M$.

REMARK. In particular, the Kobayashi pseudo-metric is a metric on $T(M)$. 
Definition 2.4. A holomorphic motion of a set $E \subset \widehat{\mathbf{C}}$ is a mapping $f: \mathbf{D} \times E \rightarrow \widehat{\mathbf{C}}$ which satisfies

(1) $f(0, z)=z$ for all $z \in E$

(2) the map $z \mapsto f(t, z)$ is injective for all $t \in \mathbf{D}$

(3) for each $z \in E$, the mapping $t \mapsto f(t, z)$ is holomorphic in $t \in \mathbf{D}$

The holomorphic motions were introduced by Mañé, Sad and Sullivan [41]. They proved the following important property of holomorphic motions of $\mathbf{C}$.

TheOrem 2.4. Let $f: \mathbf{D} \times \widehat{\mathbf{C}} \rightarrow \widehat{\mathbf{C}}$ be a holomorphic motion. Then $f_{t}=$ $f(t, \cdot)$ is a quasiconformal map for each $t \in \mathbf{D}$ such that the quasiconformal constant $K(t)$ of $f_{t}$ satisfies $K(t) \leq \frac{1+|t|}{1-|t|}$. In addition, the map $t \rightarrow \mu_{t}$ is a holomorphic map from $\mathbf{D}$ onto the unit ball $U$ of $L^{\infty}(\mathbf{D})$ where $\mu_{t}$ is the Beltrami coefficient of $f_{t}$.

A natural question was whether a holomorphic motion of a subset of $\widehat{\mathbf{C}}$ extends to a holomorphic motion of $\widehat{\mathbf{C}}$. This was positively answered by Slodkowski [57].

ThEOREM 2.5. Let $f: \mathbf{D} \times E \rightarrow \widehat{\mathbf{C}}$ be a holomorphic motion of a closed subset $E$ of $\widehat{\mathbf{C}}$. Then the holomorphic motion $f$ of $E$ extends to a holomorphic motion of $\widehat{\mathbf{C}}$.

Earle, Kra, and Krushkal [17] obtained a group invariant version of the Slodkowski's Extension Theorem as follows.

THEOREM 2.6. Let $f: \mathbf{D} \times E \rightarrow \widehat{\mathbf{C}}$ be a holomorphic motion of a closed set $E \subset \widehat{\mathbf{C}}$ which contains at least three points. Let $G$ be a group of Möbius maps which setwise preserve $E$. If for each $\gamma \in G$ and $t \in \mathbf{D}$ there exists a Möbius map $\gamma_{t}$ such that

$$
f(t, \gamma(z))=\gamma_{t}(f(z, t))
$$

for $z \in E$ and $t \in \mathbf{D}$, then $f$ can be extended to a holomorphic motion of $\widehat{\mathbf{C}}$ which also satisfies the above invariance property.

Earle, Kra, and Krushkal [17] used Theorem 2.6 to give a new and unified proof of Royden's theorem that the Kobayashi metric on $T(M)$ is equal to the Teichmüller metric. Other applications of the invariant extensions of holomorphic motions are discussed in the next section. For further study and applications of holomorphic motions see $[43],[44]$. 
2.5. The tangent space to the Teichmüller space. The Bers embedding (see Theorem 2.1) shows that the Teichmüller space $T(M)$ of a hyperbolic surface $M$ is embedded in the Banach space $B_{\mathbf{L}}(G)$ as an open bounded subset. This embedding provides a global holomorphic chart for $T(M)$. Thus the tangent space at the basepoint of $T(M)$ is identified with $B_{\mathbf{L}}(G)$.

Let $[f] \in T(M)$ and let $\mu$ be the Beltrami coefficient of $f$. Since $\mu$ satisfies (2), it follows that $f$ conjugates $G$ onto another Fuchsian group $G^{\mu}$. We noted that the translation map $T(\mu): T\left(M^{\mu}\right) \rightarrow T(M)$ is biholomorphic. (Recall that the translation map $T(\mu)$ sends the basepoint of $T\left(M^{\mu}\right)$ to the point $[f] \in T(M)$.) Thus the tangent space at $[f] \in T(M)$ is isomorphic to the tangent space at the basepoint of $T\left(M^{\mu}\right)$.

The Teichmüller space $T(M)$ is defined as a quotient of the open unit ball $U(G)$ in $L^{\infty}(G)$ with respect to the relation (2). We also note that the Schwarzian derivative map $\mathcal{S}: U(G) \rightarrow B_{\mathbf{L}}(G)$ is holomorphic. This follows from the measurable Riemann mapping theorem (see Ahlfors and Bers [4]). This implies that a differentiable path $t \mapsto \mu_{t}$ in $U(G)$ projects to a differentiable path $t \mapsto \mathcal{S}\left(\mu_{t}\right)$ in $B_{\mathbf{L}}(G)$. The Ahlfors-Weil section gives a holomorphic section $\mathbf{s}$ of the Schwarzian map $\mathcal{S}: U(G) \rightarrow B_{\mathbf{L}}(G)$ from a neighborhood of the basepoint in $T(M)$ into $U(G)$. Thus, a differentiable path through a neighborhood of the origin in $B_{\mathbf{L}}(G)$ lifts to a differentiable path in $U(G)$ through the origin. Since the derivative of a differentiable path in $U(G)$ gives an element in $L^{\infty}(G)$, we conclude that each Beltrami differential $\mu \in L^{\infty}(G)$ represents a tangent vector at the basepoint in $T(M)$, and conversely each tangent vector at the basepoint of $T(M)$ is represented by some $\mu \in L^{\infty}(G)$. A single tangent vector is represented by many Beltrami differentials. We denote by $[\mu]_{\text {tan }}$ the class of all Beltrami differentials which represent the same tangent vector as $\mu \in L^{\infty}(G)$. (Recall that for $\mu \in U(G)$, we denote by $[\mu]$ its Teichmüller class, i.e., the point in $T(M)$ represented by $\mu$.)

The Bers embedding and the Bers reproducing formula $[\mathbf{6}]$ provide the criteria for two Beltrami differentials to represent the same tangent vector at the basepoint of $T(M)$. Let $M=\mathbf{H} / G$ and let $\omega$ be a fundamental polygon for $G$ in $\mathbf{H}$. We denote by $B_{\mathbf{H}}(G)$ the space of all holomorphic functions $\psi: \mathbf{H} \rightarrow \mathbf{C}$ which satisfy the condition (3) in $\mathbf{H}$. Let

$$
A(G)=\left\{\phi \in B_{\mathbf{H}}(G):\|\phi\|_{L^{1}(\omega)}=\iint_{\omega}|\phi(z)| d x d y<\infty\right\}
$$

and let

$$
N(G)=\left\{\mu \in L^{\infty}(G): \iint_{\omega} \mu(z) \phi(z) d x d y=0 \text { for all } \phi \in A(G)\right\} .
$$

The following is a theorem of Ahlfors-Bers.

Theorem 2.7. Let $M$ be a hyperbolic Riemann surface and let $G$ be a Fuchsian group such that $M=\mathbf{H} / G$. Then the Schwarzian derivative map 
$\mathcal{S}: U(G) \rightarrow T(M)$ has a Fréchet derivative $\dot{\mathcal{S}}=P$ which is a bounded, linear projection map $P: L^{\infty}(G) \rightarrow B_{\mathbf{L}}(G)$ given by

$$
P(\mu)(z)=-\frac{6}{\pi} \iint_{\mathbf{H}} \frac{\mu(\zeta)}{(\zeta-\bar{z})^{4}} d \eta d \xi .
$$

The kernel ker $(P)$ of the projection map $P$ is $N(G)$. This implies that $P$ induces a linear isomorphism

$$
\bar{P}: L^{\infty}(G) / N(G) \rightarrow B_{\mathbf{L}}(G) .
$$

REMARK. Let $\mu \in L^{\infty}(G)$. The above theorem states that $[\mu]_{\text {tan }}=\mu+$ $N(G)$.

2.6. The Douady-Earle extension. Every quasiconformal homeomorphism $f: \mathbf{H} \rightarrow \mathbf{H}$ extends continuously to a homeomorphism of $\widehat{\mathbf{R}}$, and this extension is a quasisymmetric map [3]. Conversely, a quasisymmetric map of $\widehat{\mathbf{R}}$ extends to a quasiconformal map of $\mathbf{H}[\mathbf{1 0}]$. This shows that there is a bijection between the Teichmüller space $T(\mathbf{H})$ of the upper halfplane $\mathbf{H}$ (called the universal Teichmüller space) and the space of all quasisymmetric maps of $\widehat{\mathbf{R}}$ up to an equivalence, where two quasisymmetric maps $h_{1}, h_{2}: \widehat{\mathbf{R}} \rightarrow \widehat{\mathbf{R}}$ are equivalent if there exists $\beta \in P S L_{2}(\mathbf{R})$ such that $h_{1}=\beta \circ h_{2}$.

Let $M$ be a hyperbolic surface and let $G$ be a Fuchsian group such that $M=\mathbf{H} / G$. Then the Teichmüller space $T(M)$ embeds into the space of quasisymmetric maps of $\widehat{\mathbf{R}}$ up to the above equivalence and the quasisymmetric maps $h: \widehat{\mathbf{R}} \rightarrow \widehat{\mathbf{R}}$ in the image satisfy the invariance property

$$
h \circ \gamma \circ h^{-1} \in P S L_{2}(\mathbf{R})
$$

for all $\gamma \in G$. To show that every quasisymmetric map which satisfies (11) is in the image of $T(M)$, it was needed to find a quasiconformal extension of quasisymmetric maps which satisfy the invariance property (11) in $\mathbf{H}$. This was achieved by Douady and Earle [11] and their extension enjoys many other important properties. We denote by Homeo $(\widehat{\mathbf{R}})$ and Homeo $(\mathbf{H})$ the space of orientation preserving homeomorphisms of the extended real line $\hat{\mathbf{R}}$ and the space of orientation preserving homeomorphisms of the upper half-plane $\mathbf{H}$, respectively.

TheOrem 2.8. There exists a map ex $\operatorname{Homeo}(\widehat{\mathbf{R}}) \rightarrow \operatorname{Homeo}(\mathbf{H})$ with the following properties:

(1) $e x(i d)=i d$,

(2) $\left.\operatorname{ex}(h)\right|_{\widehat{\mathbf{R}}}=h \quad$ for $h \in \operatorname{Homeo}(\widehat{\mathbf{R}})$,

(3) $e x(h)$ and $e x(h)^{-1}$ are real analytic,

(4) if $\alpha, \beta \in P S L_{2}(\mathbf{R})$ then ex $(\alpha \circ h \circ \beta)=\alpha \circ$ ex $(h) \circ \beta$ (the conformal naturality),

(5) if $h$ is quasisymmetric then ex $(h)$ is quasiconformal. 
REMARK. One can construct the barycentric extension by using certain dynamical systems on the unit disc (see [1]). For the barycentric extensions of monotone maps see [2].

The barycentric map $b: U(\mathbf{H}) \rightarrow U(\mathbf{H})$ is defined as follows. For $\mu \in$ $U(\mathbf{H})$ we denote by $f^{\mu}$ a quasiconformal map of $\mathbf{H}$ onto itself whose Beltrami coefficient is $\mu$. Then $b(\mu)$ is the Beltrami coefficient of $e x\left(\left.f^{\mu}\right|_{\widehat{\mathbf{R}}}\right)$. The following theorem states further properties of the barycentric extension.

THEOREM 2.9. The barycentric map $b: U(\mathbf{H}) \rightarrow U(\mathbf{H})$ satisfies the following properties:

(1) The Beltrami coefficient $b(\mu)$ is a real analytic function on $\mathbf{H}$,

(2) $b(\mu)=b(\nu)$ if and only if $\mu$ and $\nu$ are Teichmüller equivalent,

(3) $\left.f^{b(\mu)}\right|_{\widehat{\mathbf{R}}}=\left.\beta \circ f^{\mu}\right|_{\widehat{\mathbf{R}}}$ for some $\beta \in P S L_{2}(\mathbf{R})$,

(4) $b(b(\mu))=b(\mu)$.

The above theorem has an immediate corollary for the topology of general Teichmüller spaces.

Corollary 2.1. The Teichmüller space $T(M)$ of a hyperbolic surface $M$ is contractible.

REMARK. The contractibility for Teichmüller spaces of finite Riemann surfaces $M$ is a corollary of the Teichmüller Theorem 3.2. Namely, the Teichmüller space $T(M)$ of a finite Riemann surface $M$ is homeomorphic to the open unit ball in $A(G)$ (for example see [26]). However, for geometrically infinite Riemann surfaces such identification is not valid. See Section 5 and [56] for another approach to contractibility.

\section{The geometry of general Teichmüller spaces}

We consider metric properties of the Teichmüller metric on general Teichmüller spaces. Note that the Teichmüller distance between the basepoint $[i d]$ and $[f]$ is given by

$$
d([f],[i d])=\frac{1}{2} \inf _{f_{1} \in[f]} \log K\left(f_{1}\right)
$$

for $[f],[i d] \in T(M)$. Since the family of normalized $K$-quasiconformal maps is compact for the uniform convergence on compact sets, it follows that the infimum in the definition of the Teichmüller metric is achieved for some map $f_{*} \in[f]$. We call the map $f_{*}$ an extremal map. An extremal map has the smallest quasiconformal dilatation among all maps homotopic to $f$, and it is not necessarily a unique map with this property. 
3.1. Extremal maps and geodesics. By the above remarks, the Teichmüller distance between the basepoint $[i d]$ and any other point $[f] \epsilon$ $T(M)$ is given by $1 / 2 \log K\left(f_{*}\right)$, where $f_{*}$ is an extremal map. Let $\mu_{*}$ be the Beltrami coefficient of the extremal map $f_{*}$. Then $K\left(f_{*}\right)=\frac{1+\left\|\mu_{*}\right\|_{\infty}}{1-\left\|\mu_{*}\right\|_{\infty}}$ and $\mu_{*}$ has the smallest essential supremum norm (i.e., $\left\|\mu_{*}\right\|_{\infty}$ is smallest) among all Beltrami coefficients in the Teihmüller class $[\mu]$ of $\mu$. The Beltrami coefficient $\mu_{*}$ is called extremal Beltrami coefficient.

For $|t|<1 /\left\|\mu_{*}\right\|_{\infty}, t \in \mathbf{R}$, we have that $t \mu_{*} \in U(G)$, namely $\left\|t \mu_{*}\right\|_{\infty}<1$. Using the chain rule and the fact that the Teichmüller distance resembles the hyperbolic distance in the upper half-plane, it easily follows that $t \mu_{*}$ is extremal for $|t|<1 /\left\|\mu_{*}\right\|_{\infty}, t \in \mathbf{R}$ (see [38]). Moreover, the path $t \mapsto\left[t \mu_{*}\right]$ for $|t|<1 /\left\|\mu_{*}\right\|_{\infty}, t \in \mathbf{R}$, is a geodesic for the Teichmüller metric (see [38]). An important question is to determine which maps in a given Teichmüller class are extremal.

3.2. The Teichmüller theorem and the Reich-Strebel inequality. Let $\varphi \in A(G)$. The Beltrami coefficient $k \frac{|\varphi|}{\varphi}$, for $-1<k<1$, is said to be of Teichmüller type. A quasiconformal map whose Beltrami coefficient is of Teichmüller type is said to be Teichmüller map. A celebrated result of Teichmüller states that any homotopy class of a quasiconformal map from one closed Riemann surface onto another closed Riemann surface contains a unique extremal map which is a Teichmüller map. A corollary of the Teichmüller theorem is that any two points in the Teichmüller space of a closed surface are connected by a unique geodesic and that the Teichmüller space is homeomorphic to a unit ball in the Euclidean space. The same results hold for Teichmüller space of finite Riemann surfaces. The methods of proof that Teichmüller used do not easily extend to geometrically infinite Riemann surfaces. The modern proof of the Teichmüller's theorem and its extension to geometrically infinite Riemann surfaces rests on the work of Reich and Strebel $[\mathbf{4 9}]$ (see also $[\mathbf{2 6}],[\mathbf{3 1}]$ ).

TheOREM 3.1 (Reich-Strebel inequality). Let $M$ be a hyperbolic surface and let $G$ be a Fuchsian group such that $M=\mathbf{H} / G$. Let $\omega \subset \mathbf{H}$ be a fundamental polygon for $G$. Let $\mu \in L^{\infty}(G)$ be a Beltrami coefficient which is Teichmüller equivalent to the trivial Beltrami coefficient 0, namely the quasiconformal map $f^{\mu}: \mathbf{H} \rightarrow \mathbf{H}$ whose Beltrami coefficient is $\mu$ is equal to a Möbius map on $\widehat{\mathbf{R}}$. Then

$$
\iint_{\omega}|\varphi(z)| d x d y \leq \iint_{\omega} \frac{\left|1+\mu(z) \frac{\varphi(z)}{|\varphi(z)|}\right|^{2}}{1-|\mu(z)|^{2}}|\varphi(z)| d x d y,
$$

for $\varphi \in A(G)$.

The Reich-Strebel inequality applies to a Beltrami coefficient which is Teichmüller trivial, namely the normalized quasiconformal map of $\mathbf{H}$ with 
this Beltrami coefficient is the identity on $\widehat{\mathbf{R}}$. Let $f$ and $g$ be two Teichmüller equivalent quasiconformal maps. The Beltrami coefficient $\mu\left(f \circ g^{-1}\right)$ of $f \circ g^{-1}$ is Teichmüller equivalent to the trivial Beltrami coefficient 0 and the ReichStrebel inequality applies to $\mu\left(f \circ g^{-1}\right)$. If the Beltrami coefficient $\mu(f)$ is of Teichmüller type, then the Reich-Strebel inequality combined with the chain rule gives the uniqueness part of the Teichmüller theorem.

THEOREM 3.2. Let $M$ be a hyperbolic Riemann surface and let $G$ be a Fuchsian group such that $M=\mathbf{H} / G$. Let $f: \mathbf{H} \rightarrow \mathbf{H}$ be a quasiconformal map which satisfies (1). If $f$ is a Teichmüller map then $f$ is a uniquely extremal map in its Teichmüller class. Moreover, if $M$ is either closed or finite Riemann surface then every homotopy class contains a unique Teichmüller map.

3.3. The Finsler metric. The Reich-Strebel inequality is used to describe the Finsler structure of the Teichmüller metric. If $\mu \in L^{\infty}(G)$ represents a tangent vector at the basepoint of $T(M)$, then the infinitesimal form for Teichmüller metric is given by

$$
|\mu|_{T_{*}(T(M))}=\sup _{\varphi}\left|\operatorname{Re} \iint_{\omega} \mu(z) \varphi(z) d x d y\right|
$$

where the supremum is over all $\varphi$ in the unit sphere in $A(G)$ and $\omega$ is a fundamental polygon for the action of $G$.

3.4. The embedding of a general Teichmüller space into the universal Teichmüller space. The universal Teichmüller space $T(\mathbf{H})$ is the Teichmüller space of the upper half-plane $\mathbf{H}$. In this case the group $G=\{i d\}$ is trivial. By the Definition 2.1, $T(\mathbf{H})$ consists of equivalence classes of quasiconformal maps $f: \mathbf{H} \rightarrow \mathbf{H}$, where two maps $f_{1}$ and $f_{2}$ are equivalent if there exists $\beta \in P S L_{2}(\mathbf{R})$ such that $\left.f_{1}\right|_{\widehat{\mathbf{R}}}=\left.\beta \circ f_{2}\right|_{\widehat{\mathbf{R}}}$.

If $M=\mathbf{H} / G$ then $T(M)$ consists of all quasiconformal maps of $\mathbf{H}$ which satisfy the invariance property (1) modulo the equivalence relation. Since the equivalence relation for $T(\mathbf{H})$ restricts to the equivalence relation for $T(M)$, it follows immediately that $T(M)$ embeds as a proper closed subset of $T(\mathbf{H})$.

Let $[f],[g] \in T(M) \subset T(\mathbf{H})$. The Teichmüller distance $d_{T(M)}([f],[g])$ between $[f]$ and $[g]$ in $T(M)$ is inf $\log K\left(g_{1} \circ f_{1}^{-1}\right)$ where the infimum is taken with respect all $f_{1}$ and $g_{1}$ such that $\left.f_{1}\right|_{\widehat{\mathbf{R}}}=\left.f\right|_{\widehat{\mathbf{R}}}$ and $\left.g_{1}\right|_{\widehat{\mathbf{R}}}=\left.g\right|_{\widehat{\mathbf{R}}}$, and both $f_{1}$ and $g_{1}$ satisfy the invariance property (1). The Teichmüller distance in $T(\mathbf{H})$ is inf $\log K\left(g_{1} \circ f_{1}^{-1}\right)$ where the infimum is over all quasiconformal maps $f_{1}, g_{1}$ satisfying $\left.f_{1}\right|_{\widehat{\mathbf{R}}}=\left.f\right|_{\widehat{\mathbf{R}}}$ and $\left.g_{1}\right|_{\widehat{\mathbf{R}}}=\left.g\right|_{\widehat{\mathbf{R}}}$ with no requirement on the invariance. We immediately obtain that

$$
d_{T(\mathbf{H})}([f],[g]) \leq d_{T(M)}([f],[g])
$$

for all $[f],[g] \in T(M)$. 
Recall that the Teichmüller metric is a Finsler metric given as follows. The norm of any Beltrami differential $\mu \in L^{\infty}(G)$ (representing a tangent vector) is defined by

$$
\|\mu\|_{T_{*}(T(M))}=\sup _{\|\varphi\|_{L^{1}(\omega)}=1}\left|\operatorname{Re} \iint_{\omega} \mu(z) \varphi(z) d x d y\right|
$$

where $\omega$ is a fundamental polygon for $G$ and $\varphi \in A(G)$. For the universal Teichmüller space we take the supremum over all holomorphic functions $\varphi$ which are integrable on $\mathbf{H}$ and of unit norm. Denote by $A$ the space of integrable holomorphic functions on $\mathbf{H}$.

There is a mapping $\Theta: A \rightarrow A(G)$ given by the Poincaré theta series

$$
\Theta(\varphi)=\sum_{\gamma \in G}(\varphi \circ \gamma)\left(\gamma^{\prime}\right)^{2}
$$

for $\varphi \in A$.

Theorem 3.3. Let $G$ be a Fuchsian group acting on $\mathbf{H}$. The Poincaré theta series defines a continuous surjective linear operator from $A$ onto $A(G)$ whose norm is at most one. Moreover, the image of the unit ball in $A$ contains a ball of radius $1 / 3$ in $A(G)$.

For $\varphi \in A$ and $\mu \in L^{\infty}(G)$, we have that $\iint_{\mathbf{H}} \mu(z) \varphi(z) d x d y=\iint_{\omega} \mu(z) \Theta$ $(\varphi)(z) d x d y$. The above theorem implies the inequality

$$
d_{T(M)}([f],[g]) \leq 3 d_{T(\mathbf{H})}([f],[g])
$$

for all $[f],[g] \in T(M) \subset T(\mathbf{H})$.

It was a conjecture of Kra $[35]$ that the norm $\|\Theta\|$ of the Poincaré theta series is strictly less that 1 if $M$ is a finite Riemann surface. A more general statement was proved by McMullen [45] (see also [5] and [46]).

TheOREM 3.4. Let $\pi: Y \rightarrow X$ be a covering of a hyperbolic surface $X$ and let $\Theta: A(Y) \rightarrow A(X)$ be the Poincaré theta series. Then either:

(1) The covering is amenable, and the image under $\Theta$ of the unit ball in $A(Y)$ is the unit ball in $A(X)$, or

(2) The covering is nonamenable, and the closure of the image of the unit ball in $A(Y)$ is contained in the interior of the unit ball of $A(X)$

If $X$ is a finite Riemann surface then either:

(1) The covering is amenable, $\|\Theta\|=1$ and the inclusion $T(X) \subset T(Y)$ is isometry for the Teichmüller metrics, or

(2) The covering is nonamenable, $\|\Theta\|<1$ and the inclusion $T(X) \subset$ $T(Y)$ is a contraction.

REMARK. It follows that when $X$ is a finite Riemann surface and $Y=\mathbf{H}$ the inclusion $T(X) \hookrightarrow T(\mathbf{H})$ is a contraction. More precisely, $d_{T(\mathbf{H})}([f],[g])<$ $d_{T(X)}([f],[g])$ if and only if $\left.f \circ g^{-1}\right|_{\widehat{\mathbf{R}}}$ is not in $P S L_{2}(\mathbf{R})$. 
3.5. Conditions for extremality. If $M$ is a geometrically infinite Riemann surface then not every homotopy class contains a Teichmüller map. The first example of a homotopy class of a quasiconformal map of $\mathbf{H}$ which does not contain a Teichmüller map is given by Strebel [58] (see also [38]). However, Strebel [59] gave a sufficient condition for a homotopy class to contain a Teichmüller map. Let $C$ be a compact subset $M$ and let $\tilde{C}$ be the lift of $C$ to $\mathbf{H}$. Let $f: \mathbf{H} \rightarrow \mathbf{H}$ be a quasiconformal map. Denote by $K_{z}(f)$ the quasiconformal dilatation of $f$ at point $z \in \mathbf{H}$. (Note that $K_{z}(f)$ is defined for almost all $z \in \mathbf{H}$ for a fixed $f$.) Define $H_{\tilde{C}}(f)=\left\|\left.K_{z}(f)\right|_{\mathbf{H} \backslash \tilde{C}}\right\|_{\infty}$.

Theorem 3.5 (The Frame Mapping Condition). Let $M$ be a geometrically infinite Riemann surface and let $[f] \in T(M)$. Let $K_{0}$ be the dilatation of an extremal map $f_{0} \in[f]$. If there exists a compact set $C \subset M$ and $f_{1} \in[f]$ such that $H_{\tilde{C}}\left(f_{1}\right)<K_{0}$ then the homotopy class $[f]$ of $f$ contains a Teichmüller map, where $\tilde{C}$ is the lift of $C$ to $\mathbf{H}$.

REMARK. The mapping $f_{1} \in[f]$ such that $H_{\tilde{C}}\left(f_{1}\right)<K_{0}$ is called the frame mapping.

A point $[f]$ in the Teichmüller space $T(M)$ is called a Strebel point if it contains a frame mapping. The set of Strebel points in $T(M)$ is dense and open (see $[\mathbf{1 8}],[\mathbf{3 6}]$ ).

Observe that each $\mu \in L^{\infty}(G)$ acts as a bounded linear functional on $A(G)$. In fact, the dual $A^{*}(G)$ of $A(G)$ is identified with $L^{\infty}(G) / N(G)[\mathbf{3}]$, [26]. Recall that $[\mu]_{\text {tan }}$ is the coset $\mu+N(G)$ and call it the tangent class of $\mu$.

A Beltrami differential $\mu_{0} \in[\mu]_{\text {tan }}$ is said to be infinitesimally extremal if $\left\|\mu_{0}\right\|_{\infty}=\sup \left|\iint_{\omega} \mu(z) \varphi(z) d x d y\right|$ where the supremum is over all $\varphi$ in the unit sphere in $A(G)$. Another important consequence of the ReichStrebel inequality is a necessary and sufficient condition for the Teichmüller extremality.

Theorem 3.6 (Hamilton-Krushkal-Reich-Strebel). Let $M$ be a hyperbolic surface and let $G$ be a Fuchsian group such that $M=\mathbf{H} / G$. Let $\mu \in$ $U(G)$. Then $\mu$ is extremal in its Teichmüller class $[\mu]$ if and only if it is infinitesimally extremal in $[\mu]_{\text {tan }}$.

The Reich-Strebel inequality is also used in proving the principle of Teichmüller contraction (see Gardiner [28] and Earle [12]). Let $\mu \in U(G)$. Assume that $\mu_{0} \in[\mu]$ is a Teichmüller extremal Beltrami coefficient and that $\mu_{1} \in[\mu]_{\tan }$ is an infinitesimally extremal Beltrami differential. The Teichmüller efficiency of $\mu$ is the difference $\|\mu\|_{\infty}-\left\|\mu_{0}\right\|_{\infty}$ and the infinitesimal efficiency is $\|\mu\|_{\infty}-\sup _{\|\varphi\|_{L^{1}(\omega)}=1} \operatorname{Re} \iint_{\omega} \mu(z) \varphi(z) d x d y$, where $\omega$ is a fundamental polygon in $\mathbf{H}$ for $G$. The Teichmüller contraction principle states that the Teichmüller efficiency is biLipschitz equivalent to the infinitesimal efficiency on any ball in $U(G)$ with radius $r<1$, where the biLipschitz constant depends on $r$. 


\subsection{The unique extremality.}

Definition 3.1. A Beltrami coefficient $\mu \in U(G)$ is uniquely extremal if it is the only element in the Teichmüller class $[\mu]$ which satisfies $\|\mu\|_{\infty}=$ $\inf _{\mu_{1} \in[\mu]}\left\|\mu_{1}\right\|_{\infty}$. A Beltrami differential $\nu \in L^{\infty}(G)$ is uniquely extremal in the tangent class if it is the only differential in the tangent class $[\nu]_{\text {tan }}$ that satisfies $\|\nu\|_{\infty}=\inf _{\nu_{1} \in[\nu]_{\text {tan }}}\left\|\nu_{1}\right\|_{\infty}$.

The Teichmüller theorem states that the Teichmüller map is uniquely extremal. Strebel [58] gave a first example of an extremal map which is not a Teichmüller map (the example is not uniquely extremal). Further, Strebel [60] showed that the horizontal stretching in an infinite strip is uniquely extremal. The Beltrami differential of this map is of the form $k \frac{|\varphi|}{\varphi}$ but the holomorphic differential $\varphi(z) d z^{2}=d z^{2}$ is not integrable. According to our definition this implies that $k \frac{|\varphi|}{\varphi}$ is not a Teichmüller Beltrami coefficient. We call it a generalized Teichmüller Beltrami coefficient. Therefore, Strebel showed that some generalized Teichmüller Beltrami coefficients are uniquely extremal. The following characterization of uniquely extremal maps was proved by Božin-Lakic-Markovic-Mateljević in [9].

Theorem 3.7. A Beltrami coefficient $\mu \in U(G)$ is uniquely extremal in its Teichmüller class $[\mu]$ if and only if it is uniquely extremal in its tangent class $[\mu]_{\text {tan }}$.

The following characterization of uniquely extremal generalized Teichmüller coefficients was also obtained in $[\mathbf{9}]$.

THEOREM 3.8. Let $\varphi \in B_{\mathbf{H}}(G)$ and $\varphi \neq 0$. Let $f$ be a quasiconformal map of $\mathbf{H}$ onto itself whose Beltrami coefficient is $k \frac{|\varphi|}{\varphi}$, for $0<k<1$. Then $f$ is uniquely extremal in its Teichmüller class if and only if there exists $\varphi_{n} \in A(G)$ which satisfies

(1) $\varphi_{n}(z)$ converges to $\varphi(z)$ uniformly on compact subsets of $\mathbf{H}$,

(2) $k\left\|\varphi_{n}\right\|_{L^{1}(\omega)}-\operatorname{Re} \iint_{\omega} \varphi_{n}(z) \mu(z) d x d y \rightarrow 0$ as $n \rightarrow \infty$.

Another important fact proved in $[\mathbf{9}]$ is that not every uniquely extremal map is a generalized Teichmüller map and moreover has not constant absolute value Beltrami coefficient.

THEOREM 3.9. Let $M$ be a hyperbolic surface that is a subset of another Riemann surface $W$ such that $W \backslash M$ has a cluster point. Let $G$ be a Fuchsian group such that $M=\mathbf{H} / G$ and let $\varphi \in A(G)$ with $\|\varphi\|_{L^{1}(\omega)}=1$. Then for every $\epsilon>0$ and for every Beltrami coefficient $\mu \in U(G)$ there exists $\nu \in U(G)$ such that

$$
\iint_{\{z \in \omega: \nu(z) \neq \mu(z)\}}|\varphi(z)| d x d y<\epsilon
$$

and $\nu$ is uniquely extremal in its Teichmüller class. 
REMARK. This theorem shows that there are many uniquely extremal maps which do not have Beltrami coefficient whose absolute value is almost everywhere constant.

3.7. The uniqueness of geodesics in the general Teichmüller space. Given $[\mu] \in T(M)$, with $[\mu] \neq[0]$, we noted that there exists at least one geodesic connecting the basepoint [0] with $[\mu]$. This geodesic is given by $t \mapsto\left[t \mu_{1}\right], 0 \leq t \leq 1$, where $\mu_{1} \in[\mu]$ is an extremal Beltrami coefficient in the Teichmüller class $[\mu]$. We also note that $t \mapsto\left[t \mu_{1}\right]$, for $-1 /\left\|\mu_{1}\right\|_{\infty}<t<$ $1 /\left\|\mu_{1}\right\|_{\infty}$, is a maximal geodesic extension of the above geodesic connecting [0] with $[\mu]$.

The following theorem is a complete characterization of points $[\mu] \epsilon$ $T(M)$ which are connected to the basepoint $[0] \in T(M)$ by a unique geodesic. This characterization is a consequence of the equivariant extensions of holomorphic motions and it was obtained by Earle-Kra-Krushkal in [17].

Theorem 3.10. Let $M$ be a hyperbolic Riemann surface and let $G$ be a Fuchsian group such that $M=\mathbf{H} / G$. Let $\mu \in U(G)$ be an extremal Beltrami coefficient on $M$ such that $[0] \neq[\mu]$. Then the following are equivalent:

(1) The Beltrami coefficient is uniquely extremal and $|\mu|=\|\mu\|_{\infty}$ almost everywhere,

(2) There is exactly one geodesic segment connecting $[i d]$ and $[\mu]$,

(3) There is exactly one holomorphic isometry $\Psi: \mathbf{D} \rightarrow T(M)$ such that $\Psi(0)=[0]$ and $\Psi\left(\|\mu\|_{\infty}\right)=[\mu]$,

(4) There is exactly one holomorphic isometry $\hat{\Psi}: \mathbf{D} \rightarrow U(G)$ such that $\hat{\Psi}(0)=0$ and $\hat{\Psi}\left(\|\mu\|_{\infty}\right)=\mu$.

REMARK. Recall that Bozin-Lakic-Markovic-Mateljevic (see [9]) constructed examples of uniquely extremal map whose Beltrami coefficients have non-constant absolute values.

The implication $(1) \Longrightarrow(2)$ is proved by $\mathrm{Z}$. Li [39]. To illustrate the main idea in the proof of this part of the theorem, we consider the Banach space $l_{\infty}$ of all bounded sequences of complex numbers with the supremum norm $\|s\|_{\infty}=\sup _{i \in \mathbb{N}}|s(i)|$. A length of a differentiable path $p:[a, b] \rightarrow l_{\infty}$ is given by $l_{[a, b]}(p)=\int_{a}^{b}\left\|p^{\prime}(t)\right\|_{\infty} d t$. A geodesic in $l_{\infty}$ is a distance minimizing path $p$ : $[a, b] \rightarrow l_{\infty}$, namely a path $p:[a, b] \rightarrow l_{\infty}$ such that $l_{\left[t, t^{\prime}\right]}(p)=\left\|p(t)-p\left(t^{\prime}\right)\right\|_{\infty}$ for all $t, t^{\prime} \in[a, b]$. Let $\underline{1} \in l_{\infty}$ be a constant sequence whose each entry is 1 . We show that there exists a unique geodesic in $l_{\infty}$ which connects the basepoint $\underline{0} \in l_{\infty}$ with $\underline{1}$. The geodesic $p:[0,1] \rightarrow l_{\infty}$ is given by $p(t)=t \cdot \underline{1}$. Let $q:[0, \overline{1}] \rightarrow l_{\infty}$ be another geodesic such that $q(0)=\underline{0}$ and $q(1)=\underline{1}$. Let $t_{0} \in$ $(0,1)$ such that $q\left(t_{0}\right) \notin p([0,1])$. After reparametrization if necessary, we have that $l_{[0, t]}(q)=t$ and $l_{[t, 1]}(q)=1-t$ for each $t \in[0,1]$. Since $q\left(t_{0}\right) \notin p([0,1])$ and $\left\|q\left(t_{0}\right)\right\|_{\infty}=t_{0}$, it follows that there exists $i_{0} \in \mathbb{N}$ such that $q\left(t_{0}\right)\left(i_{0}\right)<t_{0}$. Then $\left|q(1)\left(i_{0}\right)-q\left(t_{0}\right)\left(i_{0}\right)\right|>1-t_{0}$ which gives $\left\|q(1)-q\left(t_{0}\right)\right\|_{\infty}>1-t_{0}$. This 
is a contradiction with the parametrization of the geodesic $q$. Thus there is exactly one geodesic connecting $\underline{0}$ and $\underline{1}$.

The above theorem characterizes points in $T(M)$ which are connected to the basepoint by a unique geodesic. A related question was to characterize points in $T(M)$ which lie on a unique maximal geodesic through the basepoint. Recall that a point $[\mu] \in T(M)$ is said to be a Strebel point if it satisfies the conditions of the Strebel's Frame Mapping Condition (see Theorem 3.5). In particular, by Theorem 3.5 a Strebel point has a Teichmüller Beltrami coefficient representative. However, not every every point $[\mu]$ which has Teichmüller representative is a Strebel point. The following theorem of Earle and Li completely characterizes points which are connected to the basepoint by a unique maximal geodesic and it describes the situation when there is more than one maximal geodesic connecting the point to the basepoint.

THEOREM 3.11. Let $[\mu] \in T(M)$ be a point different from the basepoint $[0]$. Then $[\mu]$ is a Strebel point if and only if there is a unique maximal geodesic through $[\mu]$ and the basepoint $[0] \in T(M)$. Moreover, if $[\mu]$ is not a Strebel point then there exists a holomorphic isometry $\Psi$ of the unit ball $D_{\infty}$ in $l_{\infty}$ into $T(M)$ such that $\Psi\left(k_{0}([\mu]) \cdot \underline{1}\right)=[\mu]$ and $\Psi(\underline{0})=[0]$. (The isometry is with respect to the Kobayashi metrics on $D_{\infty}$ and $T(M) . k_{0}([\mu])=$ $\inf _{\mu_{1} \in[\mu]}\left\|\mu_{1}\right\|_{\infty}$ is the minimal dilatation of the Teichmüller class $[\mu]$.)

REMARK. The geometry of $D_{\infty}$ determines the geometry of its image $\Psi\left(D_{\infty}\right) \subset T(M)$. In particular, there are infinitely many geodesics through $[\mu]$ and [0] (in this case). If $\mu$ is uniquely extremal and $|\mu|$ is constant, then Earle-Kra-Krushkal theorem (Theorem 2.6) guaranties that there is only one geodesic between $[\mu]$ and $[0]$. Earle-Li theorem says that there are infinitely many maximal extensions. Another corollary of Earle-Li theorem is that there exists a simple closed geodesic through $[\mu]$ and $[0]$ whose length is four times the distance from $[\mu]$ to $[0]$.

3.8. The bi-Lipschitz structure of Teichmüller spaces. If $M$ is a finite type Riemann surface then the Teichmüller space $T(M)$ is locally bi-Lipschitz equivalent (as a metric space with respect to the Teichüller metric) to the Euclidean space of the corresponding dimension. This means that every metric ball in $T(M)$ of finite radius can be mapped by a biLipschitz homeomorphism onto a Euclidean ball. This follows from the Bers embedding theorem 2.1. Assume that $M$ is an infinite type surface. The following theorem was proved by Fletcher [25] and it shows that all infinite dimensional Teichmüller spaces are locally bi-Lipschitz equivalent.

THEOREM 3.12. Let $M$ be an infinite type Riemann surface and let $l^{\infty}$ denote the Banach space of all bounded sequences. Then $T(M)$ is locally bi-Lipschitz equivalent to $l^{\infty}$. 
This of course does not mean that every two infinite dimensional Teichmüller spaces are globally bi-Lipschitz equivalent. We have the following conjecture.

ConjeCture 3.1. Let $M$ and $N$ be two infinite type Riemann surafaces. Suppose that there exists a bi-Lipschitz map $f: T(M) \rightarrow T(N)$. Then there exists a quasiconformal map between $M$ and $N$ and $T(M)$ and $T(N)$ are isometric to each other.

\section{Biholomorphic maps between Teichmüller spaces}

The Bers embedding (see Theorem 2.1) introduces a natural complex structure on the Teichmüller space $T(M)$ of a hyperbolic Riemann surface $M$. An important problem was to classify all biholomorphic maps between any two Teichmüller spaces $T(M)$ and $T(N)$, where $N$ is also a hyperbolic Riemann surface. A particular case of this problem was to understand all biholomorphic self-maps of a Teichmüller space $T(M)$.

The mapping class group $\mathcal{M} C(M)$ of a Riemann surface $M$ is the group of all quasiconformal maps $g: M \rightarrow M$ up to homotopy. The mapping class group $\mathcal{M} C(M)$ acts on $T(M)$ by $[f] \mapsto\left[f \circ g^{-1}\right]$ for $[g] \in \mathcal{M} C(M)$ and $[f] \in$ $T(M)$. It is clear that any $[g] \in \mathcal{M} C(G)$ induces a biholomorphic map of $T(M)$ onto itself. Such biholomorphic map is said to be geometric. More generally, a biholomorphic map from $T(M)$ onto $T(N)$ which is induced by a quasiconformal map from $N$ onto $M$ is said to be geometric.

A finite Riemann surface of genus $g$ with $n$ punctures is said to be exceptional if $2 g+n \leq 4$, otherwise it is said to be non-exceptional. Royden [50] showed that for any non exceptional closed surface $S$ each biholomorphic map of $T(S)$ is geometric. Earle and Kra [15], [16] showed that each biholomorphic map of the Teichmüller space of a non exceptional finite Riemann surface is geometric and that a biholomorphic from the Teichmüller space of a finite Riemann surface onto an open subset of the Teichmüller space of another finite Riemann surface is also necessarily geometric. (In particular, the two finite Riemann surfaces are quasiconformal.)

We give an outline of Royden's proof that a biholomorphic map $\Psi$ of the Teichmüller space $T(S)$ of a closed Riemann surface $S$ is geometric. The map $\Psi: T(S) \rightarrow T(S)$ is an isometry for the Kobayashi metric because it is biholomorphic. Since the Kobayashi metric equals the Teichmüller metric on $T(S)$, it follows that $\Psi$ is also an isometry for the Teichmüller metric. The derivative map $\Psi^{\prime}: T_{[i d]}(T(S)) \rightarrow T_{\Psi([i d])}(T(S))$ of the biholomorphic map $\Psi$ gives an isometry between tangent spaces at the basepoint $[i d] \in T(S)$ and at the image point $\Psi([i d]) \in T(S)$. The tangent space $T_{[i d]}(T(S))$ at the basepoint $[i d] \in T(S)$ is isometric to the dual of the space of all integrable holomorphic quadratic differentials on the Riemann surface $S$. Since the tangent space is finite-dimensional, it follows that an isometry between two tangent spaces gives an isometry between their pre-duals. Namely, there is an 
induced isometry between the space of holomorphic quadratic differentials on the Riemann surface $S$ and the space of holomorphic quadratic differentials on the Riemann surface $\Psi([i d])(S)$.

Let $G$ be a Fuchsian group such that $\mathbf{H} / G$ is isomorphic to $S$. Let $G_{f}=$ $f G f^{-1}$ be the conjugate Fuchsian group which uniformizes the Riemann surface $\Psi([f])(S)$. We concluded above that there exists an induced linear isometry $L: A(G) \rightarrow A\left(G_{f}\right)$.

Definition 4.1. Let $G$ and $G_{1}$ be two Fuchsian groups acting on $\mathbf{H}$. An isometry $L: A(G) \rightarrow A\left(G_{1}\right)$ is said to be geometric if it is given by

$$
L(\varphi)=\theta(\varphi \circ \alpha)\left(\alpha^{\prime}\right)^{2}
$$

for some $\theta \in \mathbf{C},|\theta|=1$ and for all $\varphi \in A(G)$, where $\alpha \in P S L_{2}(\mathbf{R})$ induces a conformal map $\bar{\alpha}: \mathbf{H} / G_{1} \rightarrow \mathbf{H} / G$.

The key ingredient in Royden's proof is that a linear isometry between $\mathbf{H} / G$ and $\mathbf{H} / G_{f}$ is necessarily geometric whenever $\mathbf{H} / G$ is a non exceptional closed Riemann surface. This implies that there is a conformal map between $\mathbf{H} / G$ and $\mathbf{H} / G_{f}$ which in turn implies that the basepoint $[i d] \epsilon$ $T(S)$ is mapped by an element $\rho_{[f]} \in \mathcal{M} C(S)$ onto $[f] \in T(S)$. The element $\rho_{[f]} \in \mathcal{M} C(S)$ such that $\rho_{[f]}([i d])=[f]$ might depend on $[f]$. However, since $\mathcal{M} C(S)$ acts properly discontinuously on $T(S)$ it follows that $\rho_{[f]}=\rho$ does not depend on $[f]$ and that $\Psi=\rho$.

Earle and Kra [15] extended Royden's argument to non exceptional finite Riemann surfaces. Earle and Gardiner [13] extended all steps in the Royden's argument to arbitrary non exceptional Riemann surfaces except the fact that an arbitrary linear isometry of the spaces of integrable holomorphic quadratic differentials is necessarily geometric. They [13] also extended Royden's original argument to show that if a Riemann surface is open with finitely generated fundamental group then each isometry of the space of integrable holomorphic quadratic differentials is necessarily geometric. Lakic [37] extended this argument further to all geometrically infinite Riemann surfaces which can be holomorphically embedded into closed Riemann surfaces.

To show that biholomorphic maps of the Teichmüller spaces of all non exceptional Riemann surfaces are necessarily geometric, it remained to show that an isometry between the spaces of integrable holomorphic quadratic differentials of two Riemann surfaces (at least one of which is non exceptional) is necessarily geometric. Markovic [42] proved that each isometry of the space of integrable holomorphic quadratic differentials is geometric by using a new method independent of Royden's argument. Combining all these results together we have the following theorem.

THEOREM 4.1. Let $M$ be a hyperbolic surface of non exceptional type. Then the space of biholomorphic automorphisms Aut $(T(M))$ coincides with the mapping class group $\mathcal{M} C(M)$ of $M$. 
Proof. We sketch proof in the case of a finite Riemann surface as given in the work of Earle and Markovic [19] which applied the techniques from $[\mathbf{4 2}]$ to the finite surface case.

Let $\hat{S}, \hat{S}_{1}$ be two closed Riemann surfaces and let $E \subset \hat{S}$ and $E_{1} \subset \hat{S}_{1}$ be two finite (and possibly empty) sets. Then $S=\hat{S} \backslash E$ and $S_{1}=\hat{S}_{1} \backslash E$ are two finite Riemann surfaces. Let $L: A(S) \rightarrow A\left(S_{1}\right)$ be a C-linear isometry between spaces of integrable holomorphic quadratic differentials on $S$ and $S_{1}$.

Let $\varphi_{0}, \ldots, \varphi_{k}$ be a basis of $A(S)$. Let $\psi_{i}=L\left(\varphi_{i}\right)$ for $i=0, \ldots, k$. Let $f_{i}=\varphi_{i} / \varphi_{0}$ and $g_{i}=\psi_{i} / \psi_{0}$ be functions from $S$ and $S_{1}$ into $\hat{\mathbf{C}}$ for $i=1, \ldots, k$. Note that the functions $f_{i}$ and $g_{i}$ can have poles at the zeroes of $\varphi_{i}$ and $\psi_{i}$. Let $S^{0}=\hat{S} \backslash\left\{p \in \hat{S}: \exists f_{i}\right.$ with pole at $\left.p\right\}$. Let $S_{1}^{0}=\hat{S}_{1} \backslash\left\{p \in \hat{S}_{1}: \exists g_{i}\right.$ with pole at $p$. Then the holomorphic functions $F=\left(f_{1}, \ldots, f_{k}\right)$ and $G=\left(g_{1}, \ldots g_{k}\right)$ map $S^{0}$ and $S_{1}^{0}$ into $\mathbf{C}^{k}$. Moreover, it follows from the Riemann-Roch theorem that if $\hat{S}, \hat{S}_{1}$ are not of exceptional type then $F$ and $G$ extend to holomorphic embeddings of $\hat{S}$ and $\hat{S}_{1}$ into $\mathbf{C P}^{k}$.

Define measures $\mu$ and $\nu$ on $\hat{S}$ and $\hat{S}_{1}$ by

$$
\mu(A)=\iint_{A}\left|\varphi_{0}\right| \text { and } \nu(B)=\iint_{B}\left|\psi_{0}\right|
$$

for $A \subset \hat{S}$ and $B \subset \hat{S}_{1}$. Since $L$ is an isometry, we get that

$$
\iint_{S}\left|1+\sum_{i=1}^{k} \lambda_{i} f_{i}\right| d \mu=\iint_{S_{1}}\left|1+\sum_{i=1}^{k} \lambda_{i} g_{i}\right| d \nu
$$

for all $\left(\lambda_{1}, \ldots, \lambda_{k}\right) \in \mathbf{C}^{k}$. The above implies that

$$
\iint_{S^{0}}\left|1+\sum_{i=1}^{k} \lambda_{i} f_{i}\right| d \mu=\iint_{S_{1}^{0}}\left|1+\sum_{i=1}^{k} \lambda_{i} g_{i}\right| d \nu
$$

because $\hat{S} \backslash S_{0}$ and $\hat{S}_{1} \backslash S_{1}^{0}$ are both finite sets.

Rudin [51] showed that the above condition implies

$$
\mu\left(F^{-1}(Q)\right)=\nu\left(G^{-1}(Q)\right)
$$

for all Borel subsets $Q \subset \mathbf{C}^{k}$. Let $Q=F\left(S_{0}\right)$ in the above equation. Then we obtain

$$
\iint_{S^{0}}\left|\varphi_{0}\right|=\mu\left(S^{0}\right)=\nu\left(G^{-1}\left(F\left(S^{0}\right)\right)\right)=\iint_{G^{-1}\left(F\left(S^{0}\right)\right)}\left|\psi_{0}\right| \leq \iint_{S_{1}^{0}}\left|\psi_{0}\right|
$$

because $G^{-1}\left(F\left(S^{0}\right)\right) \subset S_{1}^{0}$. Since $L$ is an isometry, we conclude that the above inequality is equality. Therefore $G^{-1}\left(F\left(S^{0}\right)\right)$ is a subset of $S_{1}^{0}$ of full measure. It is not hard to see that $G^{-1}\left(F\left(S^{0}\right)\right)$ is closed in $\mathbf{C}^{k}$. Thus $F\left(S^{0}\right)=$ $G\left(S_{1}^{0}\right)$. The functions $F$ and $G$ extend to embeddings $\Phi: \hat{S} \rightarrow \mathbf{C P}^{k}$ and $\Psi: \hat{S}_{1} \rightarrow \mathbf{C P}^{k}$ such that $\Phi(\hat{S})=\Psi\left(\hat{S}_{1}\right)$. 
Define a holomorphic map $h: \hat{S}_{1} \rightarrow \hat{S}$ by $h=\Phi^{-1} \circ \Psi$. The restriction of $h$ to $S_{1}^{0}$ satisfies $F \circ h=G$ and $h\left(S_{1}^{0}\right)=S^{0}$. Then

$$
\frac{L\left(\varphi_{i}\right)}{L\left(\varphi_{0}\right)}=\frac{\psi_{i}}{\psi_{0}}=g_{i}=f_{i} \circ h=\frac{h^{*}\left(\varphi_{i}\right)}{h^{*}\left(\varphi_{0}\right)} .
$$

For every measurable subset $K$ of $\hat{S}_{1}$ we have

$$
\iint_{K}\left|L\left(\varphi_{0}\right)\right|=\iint_{K}\left|\psi_{0}\right|=\nu(K)=\mu(h(K))=\iint_{K}\left|h^{*}\left(\varphi_{0}\right)\right|
$$

which implies that $L\left(\varphi_{0}\right)=\theta h^{*}\left(\varphi_{0}\right)$ for some $|\theta|=1$. From $\frac{L\left(\varphi_{i}\right)}{L\left(\varphi_{0}\right)}=\frac{h^{*}\left(\varphi_{i}\right)}{h^{*}\left(\varphi_{0}\right)}$ and the above, we get

$$
L\left(\varphi_{i}\right)=\theta h^{*}\left(\varphi_{i}\right)
$$

for all $i$.

To show that $h\left(S_{1}\right)=S$, it is enough to show that punctures are mapped onto punctures. By the separation properties of integrable holomorphic quadratic differentials, for each puncture in $\hat{S}_{1}$ there is an integrable holomorphic quadratic differential on $S_{1}$ with a simple pole at the puncture. Then $S_{1}$ consists of all points in $\hat{S}_{1}$ at which every differential $L(\varphi)$ for $\varphi \in A(S)$ has finite value. On the other hand, $h^{-1}(S)$ consists of all points in $\hat{S}_{1}$ at which every differential $h^{*}(\varphi)$ for $\varphi \in A(S)$ has finite value. The equation $L\left(\varphi_{i}\right)=\theta h^{*}\left(\varphi_{i}\right)$ implies that these two sets coincide. Thus $h^{-1}(S)=S_{1}$ and this finishes the proof.

REMARK. The proof in $[\mathbf{4 2}]$ in the case of a general Riemann surface requires additional arguments then the above proof for finite Riemann surfaces because in general there is no holomorphic embedding of a geometrically infinite surface into $\mathbf{C P}^{k}$, for any finite $k$. Thus Rudin's theorem cannot be immediately applied to construct the corresponding holomorphic map.

\section{Earthquakes and Thurston boundary for general Teichmüller spaces}

5.1. Earthquakes. Earthquakes, introduced by Thurston [63], are maps of the upper half-plane $\mathbf{H}$ onto itself. (We define left earthquakes and right earthquakes can be defined analogously. All properties of left earthquakes hold for right earthquakes as well.)

A geodesic lamination $\lambda$ on $\mathbf{H}$ is a closed subset of $\mathbf{H}$ which is foliated by pairwise disjoint geodesics of $\mathbf{H}$. (Note that the foliation of the closed subset is a necessary part of the definition because there are closed subsets of $\mathbf{H}$ which can be foliated by disjoint geodesics in more than one way. For example, $\mathbf{H}$ can be foliated by disjoint geodesics in many ways. However, this requirement is not necessary for geodesic laminations on finite hyperbolic surfaces.) A stratum of $\lambda$ is either a geodesic from the given foliation of $\lambda$ or a connected component of $\mathbf{H} \backslash \lambda$. (Note that a connected component of $\mathbf{H} \backslash \lambda$ 
is an open hyperbolic polygon whose boundary sides are geodesics in $\lambda$. A complementary polygon can have infinitely many boundary sides. From now on, a geodesic from the foliation of $\lambda$ will be simply called a geodesic in $\lambda$.)

An (left) earthquake map $E: \mathbf{H} \rightarrow \mathbf{H}$ is a bijective map defined as follows (see $[\mathbf{6 3}]$ ). Let $\lambda$ be a geodesic lamination on $\mathbf{H}$. The map $E$ when restricted to a stratum $g$ of $\lambda$ is in $P S L_{2}(\mathbf{R})$. Moreover, for any two strata $g, g_{1}$ of $\lambda$ we require that $\left.E\right|_{g} \circ\left(\left.E\right|_{g_{1}}\right)^{-1}$ is a hyperbolic translation whose axis weakly separates $g$ and $g_{1}$, and which (weakly) translates stratum $g$ to the left as seen from $g_{1}$. (In other words, the relative displacement of $E(g)$ with respect to $E\left(g_{1}\right)$ is to the left.) The geodesic lamination $\lambda$ is called the support of $E$.

It is clear that $E(\lambda)$ is a geodesic lamination on $\mathbf{H}$. $\lambda$ is called the initial and $E(\lambda)$ is called the terminal lamination of the earthquake map $E$. The fact that all relative displacements between strata are to the left gives us a well defined transverse measure to the geodesic lamination $\lambda$ as follows. (A transverse measure on $\lambda$ is a positive Radon measure on each finite hyperbolic arc transverse to geodesics in $\lambda$ which is invariant under homotopies preserving all geodesics in $\lambda$.) Let $I$ be a closed hyperbolic arc transverse to geodesics in $\lambda$. Divide arc $I$ into $n$ consecutive subarcs of equal length by points $\left\{x_{0}, x_{1}, \ldots, x_{n}\right\}$ such that $x_{0}$ and $x_{n}$ are the endpoints of $I$. Let $g_{i}$ be the stratum of $\lambda$ which contains point $x_{i}$ and let $a_{i}$ be the translation length of $\left.E\right|_{g_{i}} \circ\left(\left.E\right|_{g_{i-1}}\right)^{-1}$. Then $\sum_{i=1}^{n} a_{i}$ is an approximation of the transverse measure $\mu$ on $I[63]$, namely

$$
\mu(I)=\lim _{n \rightarrow \infty} \sum_{i=1}^{n} a_{i} .
$$

The quantity $\mu(I)$ is independent of the choice of the division points $x_{i}$ as long as the distance between any two division points goes to 0 as $n \rightarrow \infty$ and this gives a homotopy invariant positive Radon measure on $I$ whose support is $I \cap \lambda$ (see Thurston [63]). A homotopy invariant transverse measure $\mu$ to a geodesic lamination $\lambda$ arising from an earthquake map is called an earthquake measure.

Let $\gamma \in P S L_{2}(\mathbf{R})$. If $E$ is an earthquake map whose support is a geodesic lamination $\lambda$ then $\gamma \circ E$ is also an earthquake map whose support is also $\lambda$. The earthquake measure for $E$ equals the earthquake measure for $\gamma \circ E$. Conversely, if two earthquake maps $E$ and $E_{1}$ have the same earthquake measure then they differ by a post-composition with some $\gamma \in P S L_{2}(\mathbf{R})$ (see $[63])$.

An earthquake measure is approximated by the sum of the translation lengths of the relative displacements between nearby strata. This process can be reversed to obtain an earthquake map starting from an earthquake measure. Namely, given an earthquake measure $\mu$ we fix the map $E$ to be the identity on one stratum $g_{0}$ (the base stratum). For any other stratum $g$ of $\lambda$, we connect $g_{0}$ to $g$ by a closed hyperbolic arc $I$. Choose finitely many points $\left\{x_{0}, x_{1}, \ldots, x_{n}\right\}$ on $I$ such that the distance between any two 
consecutive points goes to 0 as $n \rightarrow \infty$ (and, for simplicity, that $\mu\left(x_{i}\right)=0$ for $i=1, \ldots, n-1)$. For each $x_{i}$, we choose a hyperbolic geodesic $g_{i}$ which contains $x_{i}$ such that it is either a stratum of $\lambda$ or it is contained in a stratum of $\lambda$. We orient $g_{i}$ such that $x_{i-1}$ is to the left and $x_{i+1}$ is to the right of $g_{i}$. We approximate $\left.E\right|_{g}$ with the composition $T_{g_{1}}^{\mu\left(\left[x_{0}, x_{1}\right]\right)} \circ \cdots \circ T_{g_{n}}^{\mu\left(\left[x_{n-1}, x_{n}\right]\right)}$ of hyperbolic translations, where $T_{g}^{a}$ denotes a hyperbolic translation whose translation length is $a>0$, whose repelling fixed point is the initial point of $g$ and whose attracting fixed point is the terminal endpoint of $g$. The composition $T_{g_{1}}^{\mu\left(\left[x_{0}, x_{1}\right]\right)} \circ \cdots \circ T_{g_{n}}^{\mu\left(\left[x_{n-1}, x_{n}\right]\right)}$ converges to a well defined element of $P S L_{2}(\mathbf{R})$ as $n \rightarrow \infty$ independently of the choice of points $\left\{x_{0}, x_{1}, \ldots, x_{n}\right\}$ on $I$. Then $E: \mathbf{H} \rightarrow \mathbf{H}$ is an earthquake whose measure equals $\mu$ by the construction (see $[\mathbf{6 3}]$ ).

It is important to note that not every transverse measure to a geodesic lamination gives an earthquake map in the above sense. We give an example of such transverse measure which does not give an earthquake map. Let $\lambda$ consists of geodesics $g_{i}, i \in \mathbb{Z} \backslash \mathbb{N}$, with one endpoint at $\infty$ and the other endpoint at $i$. We define a transverse measure $\mu$ on $\lambda$ to give weight $\log 2$ to each transverse intersection with any $g_{i}$. We fix $E$ to be the identity on the stratum of $\lambda$ which is the hyperbolic half-plane bounded by $g_{0}$ and having the positive real axis on its boundary at infinity. Then $\left.E\right|_{g_{i-1}}=T_{g_{i}}^{\log 2} \circ \cdots \circ T_{g_{0}}^{\log 2}$, where $g_{i}$ are oriented from $\infty$ to $i$. This gives that $\left(\left.E\right|_{g_{i-1}}\right)(i-1)=-(1 / 2+$ $\left.1 / 2^{2}+\cdots+1 / 2^{|i|}\right)>-1$ for all $i$. This implies that $E$ is not onto because $E(\mathbf{H})$ does not contain hyperbolic half-plane whose boundary is the geodesic with endpoints $\infty$ and -1 , and which contains $(-\infty,-1) \subset \mathbf{R}$ on its boundary at infinity. Thus $E$ is not an earthquake map (see [64]). Moreover, it is possible to find an earthquake measure $\mu$ which comes from an earthquake map such that the transverse measure $\frac{1}{2} \mu$ does not give an earthquake map.

By the definition, earthquake maps displace strata relatively to the left. The freedom comes from the choice of the support geodesic lamination $\lambda$ and of the amount of the displacement, i.e., the earthquake measure. An earthquake map of $\mathbf{H}$ onto itself extends to a homeomorphism of the extended real axis $\hat{R}$ (see $[\mathbf{6 3}]$ ). A fundamental theorem of Thurston $[\mathbf{6 3}]$ is that the converse is also true.

THEOREM 5.1. Each homeomorphism of the extended real line $\hat{\mathbf{R}}$ is the restriction of an earthquake map.

If a transverse measure is supported on only finitely many geodesics then there exists an earthquake with this measure. Earthquakes with finite transverse measures are called finite earthquakes. Gardiner, $\mathrm{Hu}$ and Lakic [30] gave an alternative proof of Theorem 5.1 using a finite earthquake theorem.

TheOrem 5.2. Given $n$-tuples $\left(x_{1}, \ldots, x_{n}\right)$ and $\left(y_{1}, \ldots, y_{n}\right)$ of points on $\hat{\mathbf{R}}$ in the counterclockwise order, there exists a unique finite earthquake $E$ 
whose support consists of geodesics with endpoints in $\left(x_{1}, \ldots, x_{n}\right)$ such that $E\left(x_{i}\right)=y_{i}$, for $i=1,2, \ldots, n$.

The finite earthquake theorem is used in $[\mathbf{3 0}]$ to give an alternative proof (to the proof of Thurston) of the general earthquake theorem (Theorem 5.1). The proof in $[\mathbf{3 0}]$ is by an approximation argument where a general homeomorphism $h: \hat{\mathbf{R}} \rightarrow \hat{\mathbf{R}}$ is approximated by homeomorphisms coming from finite earthquakes such that they agree with $h$ on larger and larger finite sets of points in $\hat{\mathbf{R}}$. The finite earthquake measures converge to the earthquake measure corresponding to $h$.

Given the earthquake theorem, the question was to find which earthquake measures give quasisymmetric maps of $\hat{\mathbf{R}}$. Thurston $[\mathbf{6 3}]$ introduced the notion of a bounded earthquake measure. An earthquake measure $\mu$ is said to be bounded if $\sup _{I} \mu(I)<\infty$, where the supremum is over all hyperbolic arcs $I$ of length 1 transverse to the support of $\mu$ and $\mu(I)$ is the total mass of $\mu$ on $I$. Thurston [63] showed that any bounded transverse measure to a geodesic lamination gives rise to an earthquake. The following theorem characterizing measures which give quasisymmetric maps is first proved in [52] (and it was already suggested in [63]).

THEOREM 5.3. An earthquake $E$ extends to a quasisymmetric map of $\hat{\mathbf{R}}$ if and only if the earthquake measure $\mu$ of $E$ is bounded.

REMARK. The equivalence of the two conditions in the above theorem is proved by the use of a third condition (see [52]). If $\mu$ is a bounded earthquake measure, then $t \mu$, for $t>0$, is also a bounded earthquake measure. Then $\left.t \mapsto E^{t \mu}\right|_{\hat{\mathbf{R}}}$ is called an earthquake path for $t>0$ real, where $E^{t \mu}$ is an earthquake whose measure is $t \mu$. The third condition states that the earthquake path in the parameter $t>0$ extends to a holomorphic motion of $\hat{\mathbf{R}}$ in the complex parameter $\tau=t+i s$, for $s$ small [52]. Gardiner, $\mathrm{Hu}$ and Lakic [30] gave another proof of the above theorem by analyzing tangent vectors to earthquake paths. More recently, Epstein, Marden and Markovic $[\mathbf{2 3}]$ proved the above theorem using a method similar to [52].

Proof. We sketch a rather short proof obtained recently in [53]. Let $E$ be an earthquake on $\mathbf{H}$ whose measure is $\mu$ and let $h=\left.E\right|_{\hat{\mathbf{R}}}$. Let $\lambda$ be the support of $\mu$. Assume that $h$ is quasisymmetric map and that $\mu$ is not bounded. We obtain a contradiction as follows. Since $\mu$ is not bounded, there exists a sequence $I_{n}$ of hyperbolic arcs with length $1 / n$ such that $\mu\left(I_{n}\right) \rightarrow \infty$ as $n \rightarrow \infty$. Give an arbitrary orientation to each $I_{n}$. Let $g_{l}^{n}$ and $g_{r}^{n}$ be the leftmost and the rightmost geodesic of the support of $\mu$ which intersect $I_{n}$. Orient $g_{l}^{n}$ and $g_{r}^{n}$ such that their tangent vectors at the points of intersection with $I_{n}$ together with the tangent vectors of $I_{n}$ form a positive basis of the tangent space of $\mathbf{H}$. Let $\gamma_{n} \in P S L_{2}(\mathbf{R})$ be such that $\gamma_{n}\left(g_{l}^{n}\right)$ is the geodesic $g$ with endpoints 0 and $\infty$, and that $\gamma_{n}\left(I_{n} \cap g_{l}^{n}\right)=i$. Let $g_{n}=\gamma_{n}\left(g_{r}^{n}\right)$ be the image of $g_{r}^{n}$, and let $a_{n}$ and $b_{n}$ be the initial and the 
terminal points of $g_{n}$, respectively. Then $a_{n} \rightarrow 0$ and $b_{n} \rightarrow \infty$ as $n \rightarrow \infty$ by the choice of $\gamma_{n}$. Fix a quadruple $(0, b, \infty, d)$ such that $b>0, d<0$ and $\operatorname{cr}(0, b, \infty, d)=2$. Let $\delta_{n} \in P S L_{2}(\mathbf{R})$ be such that $E_{n}=\delta_{n} \circ E \circ \gamma_{n}^{-1}$ is the identity on the geodesic with endpoints 0 and $\infty$. Then $E_{n}$ fixes $0, \infty$ and $d$, and $E_{n}(b) \rightarrow \infty$ as $n \rightarrow \infty$. Thus $\operatorname{cr}\left(E_{n}(a, b, c, d)\right) \rightarrow \infty$ as $n \rightarrow \infty$. However, this is a contradiction with the fact that each $\left.E_{n}\right|_{\hat{\mathbf{R}}}$ has a quasiconformal extension with the same quasiconformal constant as does $\left.E\right|_{\hat{\mathbf{R}}}$.

We consider the converse. Namely, assume that $\mu$ is bounded and that $h=\left.E\right|_{\hat{\mathbf{R}}}$ is not quasisymmetric. Then there exists a sequence of quadruples $\left(a_{n}, b_{n}, c_{n}, d_{n}\right)$ on $\hat{\mathbf{R}}$ such that $\operatorname{cr}\left(a_{n}, b_{n}, c_{n}, d_{n}\right)=2$ and $\operatorname{cr}\left(h\left(a_{n}, b_{n}, c_{n}, d_{n}\right)\right) \rightarrow$ $\infty$ as $n \rightarrow \infty$. There exists $\gamma_{n} \in P S L_{2}(\mathbf{R})$ such that $\gamma_{n}\left(a_{n}, b_{n}, c_{n}, d_{n}\right)=$ $(a, b, c, d)$ for a fixed quadruple $(a, b, c, d)$ whose cross-ratio is 2 . Let $\mu_{n}=$ $\gamma_{n}(\mu)$. Since $\mu$ is bounded, it follows that all $\mu_{n}$ are bounded with the same bound as $\mu$. Then there exists a subsequence $\mu_{n_{k}}$ of $\mu_{n}$ which converges in the weak* sense on each hyperbolic arc in $\mathbf{H}$. This implies that a subsequence of properly normalized earthquakes $E_{n_{k}}$ whose measures are $\mu_{n_{k}}$ weakly converge to an earthquake $E^{*}$ of $\mathbf{H}$ whose measure $\mu^{*}$ is the weak* limit of $\mu_{n_{k}}$ (see [53]). On the other hand, $\operatorname{cr}\left(E_{n}(a, b, c, d)\right) \rightarrow \infty$ as $n \rightarrow \infty$ by our assumption. However, $\operatorname{cr}\left(E_{n_{k}}(a, b, c, d)\right) \rightarrow \operatorname{cr}\left(E^{*}(a, b, c, d)\right)$ as $n_{k} \rightarrow \infty$ because $E_{n_{k}} \rightarrow E^{*}$. This is a contradiction because $E^{*}$ is a homeomorphism.

We describe in more details the additional condition which was used in the proof of Theorem 5.3 in [52]. Let $\mathbf{H}^{3}=\{(z, t): z \in \mathbf{C}, t>0\}$ be the upper half-space equipped with the hyperbolic metric whose density is $\rho(z, t)=\frac{1}{t}$. The upper half-plane $\mathbf{H}$ isometrically embeds into $\mathbf{H}^{3}$ by specifying $\mathbf{H}=\{(z, t): z \in \mathbf{C}, \operatorname{Im}(z)=0, t>0\}$. Let $\mu_{1}$ and $\mu_{2}$ be two transverse measures on $\lambda$. Then $\mu=\mu_{1}+i \mu_{2}$ is a complex valued transverse measure with the support $\lambda$. Thurston [64], [22] defined a bending map $E^{\mu}: \mathbf{H} \rightarrow \mathbf{H}^{3}$ as follows. Fix one stratum $g$ of $\lambda$ and define $E^{\mu}$ to be the identity on this stratum. Given any other stratum $g^{\prime}$, consider a closed hyperbolic arc $I$ from $g$ to $g^{\prime}$. Let $\left\{x_{0}, x_{1}, \ldots, x_{n}\right\}$ be points of division of $I$ such that the maximum of the distance between any two consecutive points $x_{i-1}$ and $x_{i}$ goes to 0 as $n \rightarrow \infty$ and such that $\mu\left(x_{i}\right)=0$ for $i=1, \ldots, n-1$. For each interval $\left(x_{i-1}, x_{i}\right)$, let $g_{i}$ be a geodesic intersecting $\left(x_{i-1}, x_{i}\right)$ which is either a stratum of $\lambda$ or which belongs to a stratum of $\lambda$. Orient $g_{i}$ such that $I$ crosses it from the left to the right. Denote by $T_{g_{i}}^{\mu\left(x_{i-1}, x_{i}\right)}$ a Möbius map in $P S L_{2}(\mathbf{C})$ which is a loxodromic element whose oriented axis is $g_{i}$ and the translation length is $\mu\left(x_{i-1}, x_{i}\right)$. Then the composition $T_{g_{0}}^{\mu\left(x_{0}, x_{1}\right)} \circ \cdots \circ T_{g_{n}}^{\mu\left(x_{n-1}, x_{n}\right)}$ approximates $\left.E^{\mu}\right|_{g^{\prime}}($ see $[\mathbf{2 2}])$. Namely,

$$
\left.E^{\mu}\right|_{g^{\prime}}=\lim _{n \rightarrow \infty} T_{g_{0}}^{\mu\left(x_{0}, x_{1}\right)} \circ \cdots \circ T_{g_{n}}^{\mu\left(x_{n-1}, x_{n}\right)} .
$$

The bending map is a generalization of the earthquake map. The image of $\mathbf{H}$ is a bent plane in $\mathbf{H}^{3}$. The bending is along the transported support of $\mu$ 
by the earthquake $E^{\mu_{1}}$ and the angle of the bending is given by the measure $\mu_{2}$ (see $[\mathbf{2 2}],[\mathbf{6 4}]$ ). It is important to note that the bending map is assumed to be injective as well. Thus, not every measure $\mu_{2}$ will produce a bending map (not even every bounded measure).

Let $\mu$ be a bounded earthquake measure. Let $\tau \in \mathbf{C}$ be a complex parameter. Then $\tau \mu$ is a complex transverse measure and for $\tau \in \mathbf{R}, \tau>0$, we get a bounded earthquake measure. The following theorem is proved in [52].

THEOREM 5.4. Let $h$ be a homeomorphism of $\hat{\mathbf{R}}$ and let $E$ be an earthquake of $\mathbf{H}$ such that $\left.E\right|_{\hat{\mathbf{R}}}=h$. Let $\mu$ be the earthquake measure of $E$. Then the following are equivalent:

(1) $h$ is a quasisymmetric map,

(2) $\mu$ is a bounded earthquake measure,

(3) there is neighborhood $V \subset \mathbf{C}$ of the real line $\mathbf{R}$ such that the earthquake path $t \mapsto E^{t \mu} \mid \hat{\mathbf{R}}$, for $t>0$, extends to a holomorphic motion $\tau \mapsto E^{\tau \mu} \mid \hat{\mathbf{R}}$ of the extended real axis $\hat{\mathbf{R}}$ for the parameter $\tau \in V$.

Moreover, the parameter $\tau$ neighborhood of the real axis is $V=\{\tau \in \mathbf{C}$ :

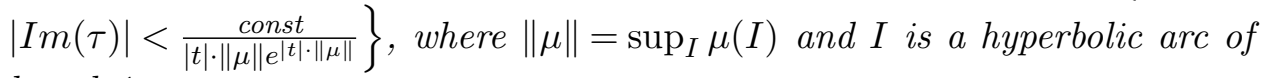
length 1 .

Remark. Epstein, Marden and Markovic [23] improved the constant to $|t| \cdot\|\mu\| e^{|t| \cdot\|\mu\|}$ from [52] where the original constant was $|t| \cdot\|\mu\| e^{8|t| \cdot\|\mu\|}$. They [23] also showed that this is the best possible constant in general.

The following is immediate consequence of the above theorem and it extends the result of Kerckhoff [34] for closed surfaces.

COROLlary 5.1. Let $\mu$ be a bounded earthquake measure. Then the earthquake path $\left.t \mapsto E^{t \mu}\right|_{\hat{\mathbf{R}}}$ is a real analytic map from the positive real axis into the universal Teichmüller space. If $\mu$ is invariant under a Fuchsian group, then the earthquake path is real analytic in $T(\mathbf{H} / G)$.

Theorem 5.4 shows that for any bounded earthquake measure $\mu$, it is always possible to bend for $\tau \in \mathbf{C}$, when $\operatorname{Im}(\tau)$ is small enough. The following theorem of Epstein, Marden and Markovic [23] considers all $\tau \in \mathbf{C}$ for which the bending map $E^{\tau \mu}$ is defined.

THEOREM 5.5. The path component containing $0 \in \mathbf{C}$ of all parameters $\tau \in \mathbf{C}$ for which the bending map $E^{\tau \mu}$ is defined is a simply connected domain in $\mathbf{C}$.

Gardiner, $\mathrm{Hu}$, and Lakic [30] considered earthquake maps which give different smoothness classes. An earthquake measure $\mu$ is said to be asymptotically trivial if $\sup _{I \subset \mathbf{H} \backslash D_{i}(n)} \mu(I) \rightarrow 0$ as $n \rightarrow \infty$, where $D_{i}(n)$ is the hyperbolic disk with center $i$ and radius $n$. For example, they $[\mathbf{3 0}]$ showed that 
an earthquake map restricts to a symmetric map of $\hat{\mathbf{R}}$ if and only if the earthquake measure is asymptotically trivial. The proof of Theorem 5.3 can be modified to obtain a short proof of the above statement (see [53]). Gardiner, $\mathrm{Hu}$, and Lakic [30], and $\mathrm{Hu}[\mathbf{3 3}]$ also obtained results about earthquake measures for earthquake maps which give $C^{1+\alpha}$ smoothness classes of homeomorphisms. Moreover, Gardiner and $\mathrm{Hu}$ showed a version of the Jackson-Zygmund approximation of the Zygmund class of functions using infinitesimal finite earthquakes (see $[\mathbf{2 9}]$ ).

5.2. Thurston boundary for general Teichmüller spaces. A celebrated Thurston boundary for the Teichmüller space of a finite hyperbolic Riemann surface $M$ is the space of projective measured laminations on $M$ [65], [64], [24]. This boundary is obtained as follows. Let $\mathcal{S}$ be the set of all homotopy classes of homotopically non-trivial and non-peripheral, simple closed curves on $M$. Given $\left[f: M \rightarrow M^{\prime}\right] \in T(M)$, we define a function $s_{[f]}$ from $\mathcal{S}$ to positive real numbers by assigning to each $\alpha \in \mathcal{S}$ the length of the geodesic representative of the curve $f(\alpha)$ on $M^{\prime}=f(M)$. The assignment $[f] \mapsto s_{[f]}$ is an embedding of the Teichmüller space $T(M)$ into the space of all positive, real functions on $\mathcal{S}$ equipped with the weak topology.

A path $t \cdot f$, for $t>0$ and $f: \mathcal{S} \rightarrow \mathbf{R}^{+}$, is said to be an asymptotic ray to $s(T(M))$ if there exists a path $s_{t} \in s(T(M))$ such that $\frac{1}{t} s_{t} \rightarrow f$ as $t \rightarrow$ $\infty$ in the weak topology. Thurston showed that an asymptotic ray to the image $s(T(M))$ of the Teichmüller space $T(M)$ is a function with special properties as follows. Namely, if $t \cdot f$, for $t>0$, is an asymptotic ray then there exists a measured lamination $\mu$ on $M$ such that $f(\alpha)=i(\alpha, \mu)$ for all $\alpha \in \mathcal{S}$. Conversely, each measured lamination induces a function $i_{\mu}$ on $\mathcal{S}$ (by using the intersection number $\left.\alpha \mapsto i_{\mu}(\alpha)=i(\mu, \alpha)\right)$ such that the ray $t \cdot i_{\mu}$, for $t>0$, is asymptotic to $s(T(M))$. Thus the boundary of $T(M)$ is identified with the space of projective measured laminations $P M L(M)$ on $M$.

Thurston's construction is putting together two different types of objects: the marked hyperbolic metrics on $M$ (obtained by taking pull-backs by the quasiconformal maps $f: M \rightarrow M^{\prime}$ ) and the projective measured laminations on $M$. Bonahon [7] unified the discussion by realizing both objects in a single space of geodesic currents. We describe a generalization (see [54], [55]) of the Bonahon's construction to general Teichmüller spaces and the corresponding Thurston boundary. From now on we resume our standing assumption that $M$ is an arbitrary hyperbolic Riemann surface.

An oriented hyperbolic geodesic in $\mathbf{H}$ is uniquely determined by its initial and terminal endpoint on $\hat{\mathbf{R}}$ and any pair of different points on $\hat{\mathbf{R}}$ determines a unique oriented geodesic. Then the space of geodesics $\mathcal{G}$ on $\mathbf{H}$ is isomorphic to $(\hat{\mathbf{R}} \times \hat{\mathbf{R}}) \backslash$ diag. The distance $d$ on $\mathcal{G}$ is defined by $d\left(g, g^{\prime}\right)=\max \{\mid a-$ $\left.a^{\prime}|| b-,b^{\prime} \mid\right\}$, where $g=(a, b), g^{\prime}=\left(a^{\prime}, b^{\prime}\right)$ and $\left|a-a^{\prime}\right|$ is the angle distance between $a$ and $a^{\prime}$ measured from $i \in \mathbf{H}$. A distance on $\mathcal{G}$ introduced by the angle distance with respect to another point in $\mathbf{H}$ is biLipschitz to $d$. A quasisymmetric map of $\hat{\mathbf{R}}$ is Hölder continuous in the angle metric. 
We define a unique positive Radon measure on the space of geodesics $\mathcal{G}$ of $\mathbf{H}$ which is invariant under the action of $P S L_{2}(\mathbf{R})$ as follows. Recall that $(\hat{\mathbf{R}} \times \hat{\mathbf{R}}) \backslash$ diag is a model for $\mathcal{G}$. The Liouville measure is by the definition

$$
L(\mathcal{A})=\iint_{\mathcal{A}} \frac{d x d y}{(x-y)^{2}}
$$

where $\mathcal{A} \subset(\hat{\mathbf{R}} \times \hat{\mathbf{R}}) \backslash$ diag is a Borel set. If $\mathcal{A}$ is a box of geodesic $[a, b] \times[c, d]$ with $[a, b] \cap[c, d]=\emptyset$, then the Liouville measure of $\mathcal{A}$ is given by $L([a, b] \times$ $[c, d])=\log c r(a, b, c, d)$, where $c r(a, b, c, d)=\frac{(c-a)(d-b)}{(d-a)(c-b)}$.

A positive Radon measure $T$ on $\mathcal{G}$ is said to be bounded if $\sup _{Q} T(Q)<\infty$ where $Q=[a, b] \times[c, d]$ is a box of geodesics and $L(Q)=\log 2$. Let $\mathcal{M}_{b}(\mathcal{G})$ be the space of all bounded measures on $\mathcal{G}$. We define the Liouville map $\mathcal{L}$ introduced by Bonahon in [7]. Recall that the universal Teichmüller space $T(\mathbf{H})$ is identified with the space of all quasisymmetric maps of $\hat{\mathbf{R}}$ modulo postcomposition with elements of $P S L_{2}(\mathbf{R})$ and that $T(M)$ is identified with the subspace of $T(\mathbf{H})$ consisting of maps which conjugate $G$ onto another Fuchsian group, where $M=\mathbf{H} / G$. Then the Liouville map

$$
\mathcal{L}: T(\mathbf{H}) \rightarrow \mathcal{M}_{b}(\mathcal{G})
$$

is defined by

$$
\mathcal{L}([h])(\mathcal{A})=h^{*}(L)(\mathcal{A})=L(h(\mathcal{A})),
$$

where $\mathcal{A} \subset \mathcal{G}$ is a Borel set and $h: \hat{\mathbf{R}} \rightarrow \hat{\mathbf{R}}$ is a quasisymmetric map.

In the case of a compact surface $M$, Bonahon and Sozen $[\mathbf{8}]$ introduced a topological vector space of Hölder distributions which contains the space of measures on the geodesics of $M$ and showed that the Liouville map is continuously differentiable in the sense of Frechét. We describe a proper generalization of the space of Hölder distributions that is adopted to a general hyperbolic Riemann surface (see [55]).

The construction and the properties of the objects related to the Liouville map $\mathcal{L}$ of the universal Teichmüller space $T(\mathbf{H})$ that we describe below are invariant under a Fuchsian group $G$ provided that we restrict $\mathcal{L}$ to the subspace of quasisymmetric maps invariant under $G$ (see [54], [55]). Therefore, our results will hold for general Teichmüller spaces. The first goal is to introduce a space containing as a subspace the space of bounded measures $\mathcal{M}_{b}(\mathcal{G})$ such that the Liouville map $\mathcal{L}$ has good smoothness properties. One candidate would be the dual space to the space of all differentiable real functions with compact support on $\mathcal{G}$. However, a conceptual problem is that such space is not invariant under the pull backs by quasisymmetric maps of $\hat{\mathbf{R}}$ (which corresponds to a change of basis for the Teichmüller space). To resolve this, note that a quasisymmetric map $h$ is Hölder continuous with the Hölder constant depending on the quasiconformal constant of the minimal quasiconformal extension of $h$. Then the space of all Hölder distributions on Hölder continuous functions is invariant under the change of base (see $[8],[55])$. 
In [55], a family of Hölder distributions in the parameter $0<\nu \leq 1$ is introduced and the intersection of the family is the space into which the universal Teichmüller space is mapped under the Liouville map. Let $0<$ $\nu \leq 1$ be fixed. For a $\nu$-Hölder continuous function $\varphi: \mathcal{G} \rightarrow \mathbf{R}$ with compact support, we define a $\nu$-norm by

$$
\|\varphi\|_{\nu}=\max \left\{\sup _{g \in \mathcal{G}}|\varphi(g)|, \sup _{g, g^{\prime} \in \mathcal{G}, g \neq g^{\prime}} \frac{\left|\varphi(g)-\varphi\left(g^{\prime}\right)\right|}{d\left(g, g^{\prime}\right)^{\nu}}\right\} .
$$

The space of $\nu$-test functions test $(\nu)$ consists of all $\nu$-Hölder continuous functions $\varphi: \mathcal{G} \rightarrow \mathbf{R}$ with support in a box $[a, b] \times[c, d]$ with $L([a, b] \times[c, d])=$ $\log 2$ such that

$$
\left\|\varphi \circ \gamma_{a, b, c, d}\right\|_{\nu} \leq 1
$$

where $\gamma_{a, b, c, d} \in P S L_{2}(\mathbf{R})$ is such that $\gamma_{a, b, c, d}:\left(a_{0}, b_{0}, c_{0}, d_{0}\right) \rightarrow(a, b, c, d)$ and $\left(a_{0}, b_{0}, c_{0}, d_{0}\right)$ is a fixed quadruple in $\hat{R}$ with $L\left(a_{0}, b_{0}, c_{0}, d_{0}\right)=\log 2$.

Let $H^{\nu}(\mathcal{G})$ be the space of all $\nu$-Hölder continuous real functions $\varphi$ : $\mathcal{G} \rightarrow \mathbf{R}$ with compact support. The space $\mathcal{H}^{\nu}(\mathcal{G})$ of $\nu$-Hölder distributions consists of all linear functionals $W: H^{\nu}(\mathcal{G}) \rightarrow \mathbf{R}$ such that

$$
\|W\|_{\nu}=\sup _{\varphi \in \operatorname{test}(\nu)}|W(\varphi)|<\infty .
$$

If $0<\nu^{\prime}<\nu$, then immediately $H^{\nu}(\mathcal{G}) \subset H^{\nu^{\prime}}(\mathcal{G})$. Let $\varphi \in H^{\nu}(\mathcal{G})$ and let $D$ be the diameter of the support of $\varphi$. Then

$$
\|\varphi\|_{\nu^{\prime}} \leq D^{\nu-\nu^{\prime}}\|\varphi\|_{\nu}
$$

This implies that

$$
\operatorname{test}(\nu) \subset D_{0}^{\nu-\nu^{\prime}} \operatorname{test}\left(\nu^{\prime}\right) \text {, }
$$

where $D_{0}$ is the diameter of $\left[a_{0}, b_{0}\right] \times\left[c_{0}, d_{0}\right]$ which is the support of each $\varphi \circ \gamma_{a, b, c, d}$. Therefore we have that

$$
\mathcal{H}^{\nu^{\prime}}(\mathcal{G}) \subset \mathcal{H}^{\nu}(\mathcal{G}) .
$$

The space $\mathcal{H}(\mathcal{G})$ of Hölder distributions is given by

$$
\mathcal{H}(\mathcal{G})=\bigcap_{0<\nu \leq 1} \mathcal{H}^{\nu}(\mathcal{G}) .
$$

Since $\mathcal{H}(\mathcal{G}) \subset \mathcal{H}^{\nu}(\mathcal{G})$ for all $0<\nu \leq 1$, it follows that $\nu$-norm on $\mathcal{H}^{\nu}(\mathcal{G})$ restricts to a norm on $\mathcal{H}(\mathcal{G})$. The family of $\nu$-norms on $\mathcal{H}(\mathcal{G})$ induces the structure of a complete metrizable topological vector space (see [55] for more details). The space $\mathcal{M}_{b}(\mathcal{G})$ of bounded measures embeds as a closed subspace in $\mathcal{H}(\mathcal{G})$ via the integration of functions in $H^{\nu}(\mathcal{G})$ against the measures.

We extended (see [55]) the target space of the Liouville map

$$
\mathcal{L}: T(\mathbf{H}) \rightarrow \mathcal{H}(\mathcal{G})
$$

The following theorem is proved in [55] (see [8] for the corresponding result for compact surfaces). 
TheOREM 5.6. The Liouville map $\mathcal{L}: T(\mathbf{H}) \rightarrow \mathcal{H}(\mathcal{G})$ is continuously differentiable in the sense of Fréchet. Namely, there exists a continuous linear map

$$
T_{\left[h_{0}\right]} \mathcal{L}: T_{\left[h_{0}\right]} T(\mathbf{H}) \rightarrow \mathcal{H}(\mathcal{G})
$$

such that if $\mathcal{B}: A \rightarrow T(\mathbf{H})$ is the inverse of the Bers embedding (with $A=$ $\Phi(T(\mathbf{H})))$ and if $\mathcal{B}\left(q_{0}\right)=\left[h_{0}\right]$, then

$$
\mathcal{L} \circ \mathcal{B}(q)=\mathcal{L} \circ \mathcal{B}\left(q_{0}\right)+T_{\left[h_{0}\right]} \mathcal{L} \circ T_{q_{0}} \mathcal{B}\left(q-q_{0}\right)+o\left(q-q_{0}\right)
$$

with $\lim _{q \rightarrow q_{0}} o\left(q-q_{0}\right) /\left\|q-q_{0}\right\|=0$ in $\mathcal{H}(\mathcal{G})$. Moreover, the tangent map $T_{\left[h_{0}\right]} \mathcal{L}$ varies continuously in $\left[h_{0}\right]$.

REMARK. Let $t \mapsto\left[h_{t}\right]$, for $t \in(-\epsilon, \epsilon)$, be a differentiable path in $T(\mathbf{H})$ with the tangent vector $v=\left.\frac{d}{d t} h_{t}\right|_{t=0}$. Let $\varphi \in H^{\nu}(\mathcal{G})$. The first step in the proof of the above theorem was to show that $\left.\frac{d}{d t} \iint \varphi d h_{t}^{*}(L)\right|_{t=0}$ exists and it equals to $T_{\left[h_{0}\right]} \mathcal{L}(v)$. The Hölder continuity of $\varphi$ is essential to have the above derivative (see [55]). Moreover, it is possible to find an explicit formula for the derivative which involves a double integration such that the order of the integration is not possible to change (see [55, Theorem 2]).

Otal [48] considered further smoothness properties of the Liouville map $\mathcal{L}: T(\mathbf{H}) \rightarrow \mathcal{H}(\mathcal{G})$. Namely, he proved

TheOREm 5.7. The Liouville map $\mathcal{L}: T(\mathbf{H}) \rightarrow \mathcal{H}(\mathcal{G})$ is real analytic.

REMARK. The proof in [48] uses a holomorphic extension of the Liouville $\mathcal{L}: T(\mathbf{H}) \rightarrow \mathcal{H}^{\nu}(\mathcal{G})$ for each $0<\nu<1$. Define $Q F(\mathbf{H})$ to be the space of equivalence classes all quasiconformal maps of the Riemann sphere $\hat{\mathbf{C}}$, where $f_{1}: \hat{\mathbf{C}} \rightarrow \hat{\mathbf{C}}$ is equivalent to $f_{2}: \hat{\mathbf{C}} \rightarrow \hat{\mathbf{C}}$ if there exists $\gamma \in P S L_{2}(\mathbf{C})$ such that $\left.f_{1}\right|_{\hat{\mathbf{R}}}=\left.\gamma \circ f_{2}\right|_{\hat{\mathbf{R}}}$. Then $T(\mathbf{H})$ is a real analytic subspace of $Q F(\mathbf{H})$ and the Liouville map extends to a holomorphic map of a neighborhood of $T(\mathbf{H})$ in $Q F(\mathbf{H})$ into the complexification $\mathcal{H}_{\mathbf{C}}^{\nu}(\mathcal{G})$ of $\mathcal{H}^{\nu}(\mathcal{G})$, for $0<\nu<1$ (see [48]). It is interesting to note that it appears that the Liouville map does not extend from a neighborhood of $T(\mathbf{H})$ in $Q F(\mathbf{H})$ into $\mathcal{H}_{\mathbf{C}}(\mathcal{G})=\bigcap_{0<\nu<1} \mathcal{H}_{\mathbf{C}}^{\nu}(\mathcal{G})$ due to the fact that the size of the neighborhood of $T(\mathbf{H})$ depends on $\nu$.

The Liouville map $\mathcal{L}: T(\mathbf{H}) \rightarrow \mathcal{H}(\mathcal{G})$ is injective. However, it turns out that the structure of the topological vector space on $\mathcal{H}(\mathcal{G})$ gives additional properties to the Liouville map (see [54] and see [7] for the closed surface case).

TheOREM 5.8. The Liouville map $\mathcal{L}: T(\mathbf{H}) \rightarrow \mathcal{H}(\mathcal{G})$ is a homeomorphism onto its image which is closed and unbounded. Moreover, the image $\mathcal{L}(T(\mathbf{H}))$ consists of all bounded, positive measures $T$ on the space of geodesics $\mathcal{G}$ which satisfy

$$
e^{-T([a, b] \times[c, d])}+e^{-T([b, c] \times[d, a])}=1
$$

for all $a, b, c, d \in \hat{\mathbf{R}}$ given in the counterclockwise order. 
An asymptotic ray to the image $\mathcal{L}(T(\mathbf{H}))$ is a path $t \mapsto t \cdot W$, for $t>0$ and $W \in \mathcal{H}(\mathcal{G})$, such that there exists a path $h_{t}^{*}(L) \in \mathcal{L}(T(\mathbf{H}))$ with

$$
\frac{1}{t} h_{t}^{*}(L) \rightarrow W
$$

as $t \rightarrow \infty$ in the topology of $\mathcal{H}(\mathcal{G})$. Therefore, it is natural to consider the set of asymptotic rays as a boundary of the Teichmüller space $T(\mathbf{H})$.

We defined a bounded measured lamination $\mu$ on $\mathbf{H}$ to be a geodesic lamination $\lambda$ (called the support of $\mu$ ) together with a transverse, homotopy invariant measure. Since the transverse measure is homotopy invariant, it follows that the transverse measure to $\lambda$ induces a bounded measure on $\mathcal{H}(\mathcal{G})$ whose support is $\lambda$. Conversely, a bounded measure on $\mathcal{H}(\mathcal{G})$ whose support $\lambda$ is a geodesic lamination induces a measured geodesic lamination on $\mathbf{H}$. For example, the Liouville measure and the pull back of the Liouville measure by any homeomorphism $h: \hat{\mathbf{R}} \rightarrow \hat{\mathbf{R}}$ are measures of full support and they do not induce measured geodesic laminations. On the other hand, a measure on $\mathcal{G}$ which is supported on finitely many non-intersecting geodesics induces a finite measured geodesic lamination.

The following result from [54] completely characterizes the Thurston boundary of general Teichmüller spaces.

TheOREM 5.9. Any asymptotic ray to $\mathcal{L}(\mathcal{G})$ is of the form $t \mapsto t \mu$, where $\mu$ is a bounded measured lamination and $t>0$. Conversely, the ray $t \mu$, for $a$ bounded measured lamination $\mu$ and $t>0$, is asymptotic to the image $\mathcal{L}\left(\left.E^{t \mu}\right|_{\hat{\mathbf{R}}}\right)$ of an earthquake path $\left.t \mapsto E^{t \mu}\right|_{\hat{\mathbf{R}}}$. Thus, the Thurston boundary of a general Teichmüller space is identified with the space of projective, bounded measured laminations.

REMARK. The hyperbolic plane does not have simple closed geodesic. An infinite Riemann surface has simple closed geodesics, but it is not always possible to parametrize the Teichmüller space using the lengths of the geodesic representatives of simple closed curves, unlike in the finite case. Thus the approach at hand using the Liouville map seems to be the correct one.

REMARK. The most demanding part of the proof of the above theorem is in establishing that the earthquake path has its projective measured geodesic lamination as its unique endpoint on the Thurston boundary. Namely, that $\frac{1}{t} \iint_{\mathcal{G}} \varphi d\left(\left.E^{t \mu}\right|_{\hat{\mathbf{R}}}\right)^{*}(L) \rightarrow \mu$ as $t \rightarrow \infty$, for all $\varphi \in H^{\nu}(\mathcal{G})$. We illustrate this convergence in the case of a simple earthquake $E$ whose earthquake measure consists of a single atom $m>0$ at the geodesic $g=(a, c)$. Let $\mu$ denote the measure with support $g$ such that $\mu(g)=m$. Then $\frac{1}{t} L\left(\left.E^{t \mu}\right|_{\hat{\mathbf{R}}}\left(\left[a^{\prime}, b^{\prime}\right] \times\left[c^{\prime}, d^{\prime}\right]\right)\right)$ converges to $m$ if $a \in\left[a^{\prime}, b^{\prime}\right]$ and $c \in\left(c^{\prime}, d^{\prime}\right]$, otherwise it converges to 0 as $t \rightarrow$ $\infty$ (see [54, Appendix, Lemma A.1]). This fact together with a more detailed information about the above convergence implies that $\frac{1}{t} \iint_{\mathcal{G}} \varphi d\left(\left.E^{t \mu}\right|_{\hat{\mathbf{R}}}\right)^{*}(L)$ $\rightarrow \mu(\operatorname{supp}(\varphi)) \cdot \varphi(g)$ as $t \rightarrow \infty$. A general case is proved by a careful analysis 
of the earthquake measure and the asymptotics of the pull backs of the Liouville measure (see [54, Section 4, Appendix] for the details).

\section{References}

[1] W. Abikoff, Conformal barycenters and the Douady-Earle extension-a discrete dynamical approach, J. Anal. Math. 86 (2002), 221-234.

[2] W. Abikoff, C. Earle and S. Mitra, Barycentric extensions of monotone maps of the circle, In the tradition of Ahlfors and Bers, III, 1-20, Contemp. Math., 355, Amer. Math. Soc., Providence, RI, 2004.

[3] L. Ahlfors, Lectures on quasiconformal mappings, Second edition. With supplemental chapters by C. J. Earle, I. Kra, M. Shishikura and J. H. Hubbard. University Lecture Series, 38. American Mathematical Society, Providence, RI, 2006.

[4] L. Ahlfors and L. Bers, Riemann's mapping theorem for variable metrics, Ann. of Math. (2) 72 (1960), 385-404.

[5] D. Barrett and J. Diller, Poincaré series and holomorphic averaging, Invent. Math. 110 (1992), no. 1, 23-27.

[6] L. Bers, Correction to "Spaces of Riemann surfaces as bounded domains", Bull. Amer. Math. Soc. 67 (1961), 465-466.

[7] F. Bonahon, The geometry of Teichmüller space via geodesic currents, Invent. Math. 92 (1988), no. 1, 139-162.

[8] F. Bonahon and Y. Sozen, Variation of the Liouville measure of a hyperbolic surface, Ergodic Theory Dynam. Systems 23 (2003), no. 3, 729-758.

[9] V. Božin, N. Lakic, V. Marković and M. Mateljević, Unique extremality, J. Anal. Math. 75 (1998), 299-338.

[10] A. Beurling and L. Ahlfors, The boundary correspondence under quasiconformal mappings, Acta Math. 96 (1956), 125-142.

[11] A. Douady and C. J. Earle, Conformally natural extension of homeomorphisms of the circle, Acta Math. 157 (1986), no. 1-2, 23-48.

[12] C. Earle, Schwarz's lemma and Teichmüller contraction, Complex manifolds and hyperbolic geometry (Guanajuato, 2001), 79-85, Contemp. Math., 311, Amer. Math. Soc., Providence, RI, 2002.

[13] C. Earle and F. Gardiner, Geometric isomorphisms between infinite-dimensional Teichmüller spaces, Trans. Amer. Math. Soc. 348 (1996), no. 3, 1163-1190.

[14] C. Earle, F. Gardiner and N. Lakic, Asymptotic Teichmüller space. II. The metric structure., In the tradition of Ahlfors and Bers, III, 187-219, Contemp. Math., 355, Amer. Math. Soc., Providence, RI, 2004.

[15] C. Earle and I. Kra, On holomorphic mappings between Teichmüller spaces, Contributions to analysis (a collection of papers dedicated to Lipman Bers), pp. 107-124. Academic Press, New York, 1974.

[16] C. Earle and I. Kra, On isometries between Teichmüller spaces, Duke Math. J. 41 (1974), 583-591.

[17] C. Earle, I. Kra and S. Krushkal, Holomorphic motions and Teichmüller spaces, Trans. Amer. Math. Soc. 343 (1994), no. 2, 927-948.

[18] C. Earle and Z. Li, Isometrically embedded polydisks in infinite dimensional Teichmüller spaces, J. Geom. Anal. 9 (1999), no. 1, 51-71.

[19] C. Earle and V. Markovic, Isometries between the spaces of $L^{1}$ holomorphic quadratic differentials on Riemann surfaces of finite type, Duke Math. J. 120 (2003), no. 2, 433-440.

[20] C. Earle, V. Markovic and D. Šarić, Barycentric extension and the Bers embedding for asymptotic Teichmüller space, Complex manifolds and hyperbolic geometry (Guanajuato, 2001), 87-105, Contemp. Math., 311, Amer. Math. Soc., Providence, RI, 2002. 
[21] C. Earle and C. McMullen, Quasiconformal isotopies, Holomorphic functions and moduli, Vol. I (Berkeley, CA, 1986), 143-154, Math. Sci. Res. Inst. Publ., 10, Springer, New York, 1988.

[22] D. Epstein and A. Marden, Convex hulls in hyperbolic space, a theorem of Sullivan, and measured pleated surfaces, Analytical and geometric aspects of hyperbolic space (Coventry/Durham, 1984), 113-253, London Math. Soc. Lecture Note Ser., 111, Cambridge Univ. Press, Cambridge, 1987.

[23] D. Epstein, A. Marden and V. Markovic, Quasiconformal homeomorphisms and the convex hull boundary, Ann. of Math. (2) 159 (2004), no. 1, 305-336.

[24] A. Fathi, F. Laudenbach and V. Poenaru, Travaux de Thurston sur les surface, Astérisque, No 66-67, Société Mathématique de France, 1979.

[25] A. Fletcher, Local rigidity of infinite-dimensional Teichmller spaces, J. London Math. Soc. (2) 74 (2006), no. 1, 26-40.

[26] F. Gardiner, Teichmüller theory and quadratic differentials, Pure and Applied Mathematics (New York). A Wiley-Interscience Publication. John Wiley \& Sons, Inc., New York, 1987.

[27] F. Gardiner, Approximation of infinite-dimensional Teichmüller spaces, Trans. Amer. Math. Soc. 282 (1984), no. 1, 367-383.

[28] F. Gardiner, On Teichmüller contraction, Proc. Amer. Math. Soc. 118 (1993), no. 3, $865-875$

[29] F. P. Gardiner and J. Hu, An earthquake version of the Jackson-Zygmund theorem, Ann. Acad. Sci. Fenn. Math. 30 (2005), no. 2, 237-260.

[30] F. Gardiner, J. Hu and N. Lakic, Earthquake curves, Complex manifolds and hyperbolic geometry (Guanajuato, 2001), 141-195, Contemp. Math., 311, Amer. Math. Soc., Providence, RI, 2002.

[31] F. Gardiner and N. Lakic, Quasiconformal Teichmüller theory, Mathematical Surveys and Monographs, 76. American Mathematical Society, Providence, RI, 2000.

[32] F. Gardiner and D. Sullivan, Symmetric structures on a closed curve, Amer. J. Math. 114 (1992), no. 4, 683-736.

[33] J. Hu, Earthquake measure and cross-ratio distortion, In the tradition of Ahlfors and Bers, III, 285-308, Contemp. Math., 355, Amer. Math. Soc., Providence, RI, 2004.

[34] S. P. Kerckhoff, Earthquakes are analytic, Comment. Math. Helv. 60 (1985), no. 1, $17-30$.

[35] I. Kra, Canonical mappings between Teichmüller spaces, Bull. Amer. Math. Soc. (N.S.) 4 (1981), no. 2, 143-179.

[36] N. Lakic, Strebel points, Lipa's legacy (New York, 1995), 417-431, Contemp. Math., 211, Amer. Math. Soc., Providence, RI, 1997.

[37] N. Lakic, An isometry theorem for quadratic differentials on Riemann surfaces of finite genus., Trans. Amer. Math. Soc. 349 (1997), no. 7, 2951-2967.

[38] O. Lehto, Univalent functions and Teichüller spaces, Graduate Texts in Mathematics, 109. Springer-Verlag, New York, 1987.

[39] L. Zhong, Nonuniqueness of geodesics in infinite-dimensional Teichm"uller spaces, Complex Variables Theory Appl. 16 (1991), no. 4, 261-272.

[40] L. Zhong, Nonuniqueness of geodesics in infinite-dimensional Teichmüller spaces, II. Ann. Acad. Sci. Fenn. Ser. A I Math. 18 (1993), no. 2, 355-367.

[41] R. Mañé, P. Sad and D. Sullivan, On the dynamics of rational maps, Ann. Sci. cole Norm. Sup. (4) 16 (1983), no. 2, 193-217.

[42] V. Markovic, Biholomorphic maps between Teichmüller spaces, Duke Math. J. 120 (2003), no. 2, 405-431.

[43] S. Mitra, Teichmüller spaces and holomorphic motions, J. Anal. Math. 81 (2000), $1-33$.

[44] S. Mitra, Teichmüller contraction in the Teichmüller space of a closed set in the sphere, Israel J. Math. 125 (2001), 45-51. 
[45] C. McMullen, Amenability, Poincaré series and quasiconformal maps, Invent. Math. 97 (1989), no. 1, 95-127.

[46] C. McMullen, Amenable coverings of complex manifolds and holomorphic probability measures, Invent. Math. 110 (1992), no. 1, 29-37.

[47] S. Nag, The complex analytic theory of Teichmüller spaces, Canadian Mathematical Society Series of Monographs and Advanced Texts. A Wiley-Interscience Publication. John Wiley \& Sons, Inc., New York, 1988.

[48] J. Otal, About the embedding of Teichmüller space in the space of geodesic Hlder distributions, Handbook of Teichmüller theory. Vol. I, 223-248, IRMA Lect. Math. Theor. Phys., 11, Eur. Math. Soc., Zürich, 2007.

[49] E. Reich and K. Strebel, On quasiconformal mappings which keep the boundary points fixed, Trans. Amer. Math. Soc. 138 (1969), 211-222.

[50] H. Royden, Automorphisms and isometries of Teichmüller space, 1971 Advances in the Theory of Riemann Surfaces (Proc. Conf., Stony Brook, N.Y., 1969) pp. 369-383 Ann. of Math. Studies, No. 66. Princeton Univ. Press, Princeton, N.J.

[51] W. Rudin, $L^{p}$-isometries and equimeasurability, Indiana Univ. Math. J. 25 (1976), no. 3, 215-228.

[52] D. Šarić, Real and complex earthquakes, Trans. Amer. Math. Soc. 358 (2006), no. 1, 233-249.

[53] D. Šarić, Bounded earthquakes, Proc. Amer. Math. Soc. 136 (2008), no. 3, 889-897 (electronic).

[54] D. Šarić, Geodesic currents and Teichmüller space, Topology 44 (2005), no. 1, 99-130.

[55] D. Sarić, Infinitesimal Liouville distributions for Teichmüller space, Proc. London Math. Soc. (3) 88 (2004), no. 2, 436-454.

[56] D. Šarić, Some remarks on bounded earthquakes, Proc. Amer. Math. Soc., 138 (2010), 871-879.

[57] Z. Slodkowski, Holomorphic motions and polynomial hulls, Proc. Amer. Math. Soc. 111 (1991), no. 2, 347-355.

[58] K. Strebel, Zur Frage der Eindeutigkeit extremaler quasikonformer Abbildungen des Einheitskreises, (German) Comment. Math. Helv. 36 1961/1962 306-323.

[59] K. Strebel, On the existence of extremal Teichmüller mappings, J. Analyse Math. 30 (1976), 464-480.

[60] K. Strebel, Eine Abschtzung der Lnge gewisser Kurven bei quasikonformer Abbildung, (German) Ann. Acad. Sci. Fenn. Ser. A. I. no. 243 (1957) 1-10.

[61] Takhtajan, L. A. and Teo, Lee-Peng, Weil-Petersson metric on the universal Teichmller space, Mem. Amer. Math. Soc. 183 (2006), no. 861, viii+119 pp.

[62] H. Tanigawa, Holomorphic families of geodesic discs in infinite-dimensional Teichmüller spaces, Nagoya Math. J. 127 (1992), 117-128.

[63] W. Thurston, Earthquakes in two-dimensional hyperbolic geometry, Low-dimensional topology and Kleinian groups (Coventry/Durham, 1984), 91-112, London Math. Soc. Lecture Note Ser., 112, Cambridge Univ. Press, Cambridge, 1986.

[64] W. Thurston, Three-dimensional geometry and topology, Vol. 1. Edited by Silvio Levy. Princeton Mathematical Series, 35. Princeton University Press, Princeton, NJ, 1997.

[65] W. Thurston, On the geometry and dynamics of diffeomorphisms of surfaces, Bull. Amer. Math. Soc. (N.S.) 19 (1988), no. 2, 417-431.

Department of Mathematics, University of Warwick, Coventry, CV8 4AL, UNITED KINGDOM

E-mail address: v.markovic@warwick.ac.uk

Department of Mathematics, Queens College of CUNy, 65-30 Kissena Blvd., FLushing, NY 11367

E-mail address: Dragomir.Saric@qc.cuny.edu 